Discrete Comput Geom 38:399-441 (2007)

DOI: $10.1007 / \mathrm{s} 00454-007-1344-5$

\title{
Solution of Scott's Problem on the Number of Directions Determined by a Point Set in 3-Space*
}

\author{
János Pach, ${ }^{1,2}$ Rom Pinchasi, ${ }^{3}$ and Micha Sharir ${ }^{2,4}$ \\ ${ }^{1}$ City College, C.U.N.Y., \\ New York, NY 10031, USA \\ ${ }^{2}$ Courant Institute of Mathematical Sciences, New York University, \\ New York, NY 10012, USA \\ pach@cims.nyu.edu \\ ${ }^{3}$ Department of Mathematics, Massachusetts Institute of Technology, \\ Cambridge, MA 02139, USA \\ ${ }^{4}$ School of Computer Science, Tel Aviv University, \\ Tel Aviv 69978, Israel \\ michas@tau.ac.il
}

\begin{abstract}
Let $P$ be a set of $n$ points in $\mathbb{R}^{3}$, not all in a common plane. We solve a problem of Scott (1970) by showing that the connecting lines of $P$ assume at least $2 n-5$ different directions if $n$ is odd and at least $2 n-7$ if $n$ is even. The bound for odd $n$ is sharp.
\end{abstract}

\section{Introduction}

According to the Gallai-Sylvester theorem [1], [6], for any set $P$ of finitely many points in the plane, not all on a line, there exists a line that passes through precisely two elements of $P$. Erdôs noticed that this result has the following corollary: Every $n$ element set $P$ with the above properties, with $n \geq 3$, determines at least $n$ connecting lines [6].

* Work on this paper by János Pach and Micha Sharir has been supported by NSF Grants CCF-05-14079 and CCR-00-98246, and by a joint grant from the U.S.-Israel Binational Science Foundation. Work by János Pach has also been supported by PSC-CUNY Research Award 63382-0032 and by Hungarian Science Foundation Grant OTKA T-032452. Work by Micha Sharir has also been supported by a grant from the Israeli Academy of Sciences for a Center of Excellence in Geometric Computing at Tel Aviv University, and by the Hermann Minkowski-MINERVA Center for Geometry at Tel Aviv University. A preliminary version of this paper has appeared in Proc. 20th Annu. ACM Sympos. Comput. Geom., 2004, pp. 76-85. Rom Pinchasi's current address is Faculty of Mathematics, The Technion, Haifa 32000, Israel; room@math.technion.ac.il. 
In 1970 Scott [16] raised the following questions: What is the minimum number of different directions assumed by the connecting lines of (1) $n$ points in the plane, not all on a line, and (2) $n$ points in 3-space, not all on a plane?

In 1982, after some initial results by Burton and Purdy [3], Ungar [18] solved the first problem, by verifying Scott's conjecture that in the plane the above minimum is equal to $2\lfloor n / 2\rfloor$, for any $n>3$. For even $n$, this result is considerably stronger than the corollary of the Gallai-Sylvester theorem mentioned above. Ungar's proof is a real gem, a brilliant application of the method of allowable sequences invented by Goodman and Pollack [8], [9]. Moreover, it solves the problem in an elegant combinatorial setting, for "pseudolines", as was suggested independently by Goodman and Pollack and by Cordovil [4]. Interestingly, there is an overwhelming diversity of extremal configurations, for which equality is attained. Four infinite families and more than one hundred sporadic configurations were catalogued by Jamison and Hill [11]. See also [10] for an excellent survey by Jamison, and the monograph of Aigner and Ziegler [1], where Ungar's proof and some of its relatives are reproduced.

In lack of a natural ordering of all directions in 3-space, Ungar's method does not seem to generalize. This explains why until recently there had not been much progress concerning Scott's second question. Scott's construction of a double pyramid whose base is a regular polygon with an even number of edges, including the center of the base (see Fig. 1), shows that the number of directions determined by $n$ noncoplanar points can be as small as $2 n-5$ if $n$ is odd. This bound was conjectured to be tight. Under the additional assumption that no three points of the set are collinear, Blokhuis and Seress [2] proved that the number of directions determined by $n \geq 6$ noncoplanar points in 3 -space is at least $7 n / 4-2$. Using the same condition, we have recently succeeded in proving the tight bound $2 n-2$ if $n$ is odd and $2 n-3$ if $n$ is even [14].

In the present paper we solve Scott's second problem in full generality (for the case of $n$ odd), by removing the assumption that no three points are collinear.

Theorem 1.1. Every set of $n \geq 6$ points in $\mathbb{R}^{3}$, not all of which are on a plane, determines at least $2 n-5$ different directions if $n$ is odd, and at least $2 n-7$ different directions if $n$ is even. This bound is sharp for every odd $n \geq 7$.

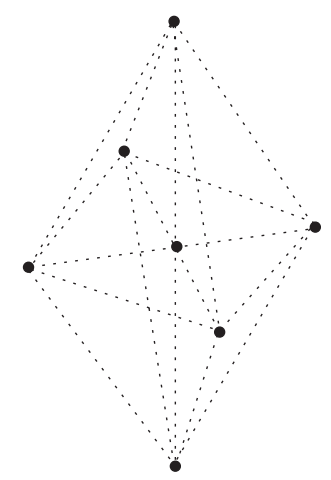

Fig. 1. A set with $n=7$ points that determines $2 n-5=9$ directions. 


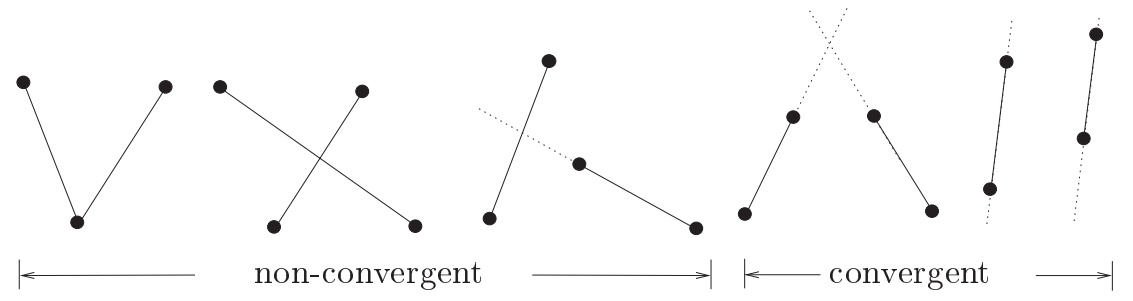

Fig. 2. Convergent and non-convergent segments.

The case where $n$ is even is handled by removing one point and applying the bound for odd $n$. Therefore, from this point on we assume that $n$ is odd. Nevertheless, we believe that the bound for even $n$ can be improved. We note that the double-pyramid construction in Fig. 1, without the center, yields an upper bound of $2 n-3$ for $n$ even.

The idea of the proof is outlined in Section 2. A key new ingredient of our argument is Theorem 3.1, proved in Section 3, which is a far reaching "bipartite" variant of Ungar's aforementioned theorem.

Definition 1.2. Two closed segments in $\mathbb{R}^{d}$ are called convergent if (i) they do not belong to the same line, and (ii) their supporting lines intersect, and the intersection point does not belong to any of the segments. See Fig. 2.

An alternative definition is that two segments are convergent if and only if they are disjoint and their convex hull is a planar quadrilateral. Two parallel segments that lie on distinct lines are also considered to be convergent (by regarding their lines to meet at infinity, or according to the alternative definition). Note also that condition (ii) rules out pairs of segments with a common endpoint.

Instead of Theorem 1.1, in Section 4 we establish the following significantly stronger result.

Theorem 1.3. Every set of $n \geq 6$ points in $\mathbb{R}^{3}$, not all of which are on a plane, determine at least $2 n-5$ segments if $n$ is odd, and at least $2 n-7$ segments if $n$ is even, no two of which are convergent and no two collinear. This bound is sharp for every odd $n \geq 7$.

We apply Theorem 1.3 in Section 5 partially to settle in the affirmative a conjecture of Blokhuis and Seress [2], showing (in Theorem 5.1) that any set $P$ of $n$ points in $\mathbb{R}^{4}$, not contained in a hyperplane and not having three collinear points, determine at least $3 n-8$ different directions, if $n$ is even, and at least $3 n-10$ different directions if $n$ is odd. The bound is sharp for every even $n \geq 8$.

Rédei's monograph on lacunary polynomials [15] was the starting point of many investigations related to algebraic variants of the above problem. For instance, it was proved in [15] that if $n$ is a prime, then any set of $n$ points in the affine plane $\operatorname{AG}(2, n)$ determines at least $(n+3) / 2$ different directions. Lovász and Schrijver [13] characterized all sets for which equality is attained. In the finite projective plane $\operatorname{PG}(2, N)$, a set $P$ of $n>4$ points, no three of which are collinear, is known to determine at least $n$ different 
directions if $N$ is odd and at least $n-1$ if $N$ is even. Equality is attained here if and only if $P$ spans a (properly defined) affinely regular $n$-gon (see [7] and [12]). The last theorem, due to Wettl [19] answers a question of Gus Simmons in cryptography. For many similar results and applications in finite geometry, algebraic number theory, and group theory, consult the survey of Szônyi [17].

\section{Preliminaries}

Let $P$ be a set of $n$ points in $\mathbb{R}^{3}$ such that not all of them lie in a common plane. Let $p_{0}$ be an extreme point of $P$, i.e., a vertex of the convex hull of $P$. Consider a supporting plane to $P$ at $p_{0}$, and translate it to the side that contains $P$. Let $\pi$ denote the resulting plane. Project from $p_{0}$ all points of $P \backslash\left\{p_{0}\right\}$ onto $\pi$. We obtain a set $R$ of points in $\pi$, not all on a line, so that each point is the image of some points of $P$. We regard $R$ as a set of weighted points, where the weight $w(r)$ of a point $r \in R$ is the number of points of $P \backslash\left\{p_{0}\right\}$ that project onto it. ${ }^{1}$ The sum of the weights is $n-1$. For a subset $A \subseteq R$, we define $w(A):=\sum_{q \in A} w(q)$.

We assume that $n$ is odd, thus $w(R)=n-1$ is even. We attempt to partition $R$ into two subsets $R^{+}, R^{-}$, so that $w\left(R^{+}\right)=w\left(R^{-}\right)=(n-1) / 2$ and all points of $R^{+}$lie to the left of every point of $R^{-}$with respect to some generic coordinate frame in $\pi$, in which no two elements of $R$ have the same $x$-coordinate.

For the choice of the coordinate frame and the partition, we begin with the following elementary geometric fact. Recall that a common inner tangent to two convex sets with disjoint interiors is a line that is tangent to both sets and separates between the interiors of the sets.

Lemma 2.1. Let $R$ be a set of non-collinear weighted points in the plane, with a total even weight $m$. Let $r$ be any vertex of the convex hull of $R$ whose weight is smaller than $m / 2$. Then one of the following properties holds:

(i) There exists a partition of $R$ into two subsets, $R^{-}$and $R^{+}$, each of overall weight $m / 2$, whose convex hulls are disjoint and which have a common inner tangent $m_{0}$ passing through $r$.

(ii) There exists a point $q \in R$ and a partition of $R \backslash\{q\}$ into two subsets, $R_{0}^{-}$and $R_{0}^{+}$, each of overall weight $<m / 2$, so that the convex hulls of $R_{0}^{-} \cup\{q\}$ and $R_{0}^{+} \cup\{q\}$ meet only at $q$, which is a common vertex of both hulls, and the line $m_{0}$ passing through $r$ and $q$ is an inner common tangent to the two hulls (supporting one of them in the edge qr).

Proof. See Fig. 3. Rotate a directed line $\ell$ counterclockwise about $r$, starting with all the points of $R \backslash\{r\}$ lying to the left of $\ell$, until the closed halfplane to the right of $\ell$ contains for the first time points with overall weight larger than $m / 2$. Let $R_{0}^{-}$denote the set $R_{0}$ of points in the open halfplane to the right of $\ell$, plus the largest set of initial

\footnotetext{
${ }^{1}$ In the preceding paper [14], where it was assumed that no three points of $P$ are collinear, $R$ was a set, or, rather, the weight of each point was 1.
} 


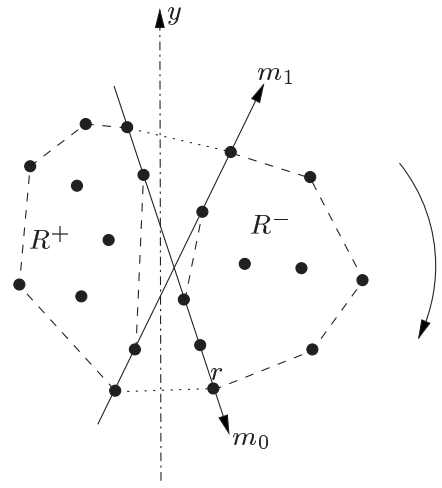

(i)

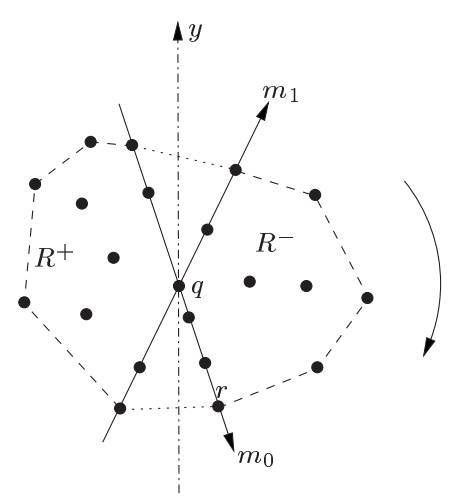

(ii)

Fig. 3. The primal construction of $R^{-}$and $R^{+}$: Cases (i) and (ii).

points of $\ell \cap R$ along $\ell$ (in their order along $\ell$ starting at $r$ ) whose overall weight does not exceed $m / 2-w\left(R_{0}\right)$.

If the overall weight of $R_{0}^{-}$is exactly $m / 2$, we are in case (i). We define $R^{-}:=R_{0}^{-}$, and $R^{+}:=R \backslash R_{0}^{-}$. See Fig. 3(i). It is clear that the convex hulls of $R^{+}$and $R^{-}$are disjoint, and that the final position of $\ell$ is the desired common inner tangent $m_{0}$.

If the overall weight of $R_{0}^{-}$is less than $m / 2$, we are in case (ii). Let $q$ be the next point of $\ell \cap R$ along $\ell$, and define $R_{0}^{+}:=R \backslash\left(R_{0}^{-} \cup\{q\}\right)$. See Fig. 3(ii). It is easily seen that the properties asserted in (ii) hold, with $m_{0}$ being the final position of $\ell$.

We apply Lemma 2.1 to our set $R \subset \pi$, with $m=n-1$. In case (ii) we split $q$ into two co-located points $q^{-}, q^{+}$, and distribute the weight $w(q)$ between them, so that $w\left(q^{-}\right)=(n-1) / 2-w\left(R_{0}^{-}\right)$and $w\left(q^{+}\right)=(n-1) / 2-w\left(R_{0}^{+}\right)$. We set $R^{-}:=R_{0}^{-} \cup\left\{q^{-}\right\}$ and $R^{+}:=R_{0}^{+} \cup\left\{q^{+}\right\}$. We refer to $q$ as the central bichromatic point of $R$.

Let $m_{1}$ denote the other inner tangent of the convex hulls of $R^{-}$and $R^{+}$. In case (ii), $m_{1}$ also passes through $q$ and through at least one other point of one of the two sets. Now choose in $\pi$ an orthogonal $(x, y)$-coordinate system whose $y$-axis is either a line that strictly separates $R^{-}$and $R^{+}$in case (i), or a line through $q$ that strictly separates $R_{0}^{-}$ and $R_{0}^{+}$in case (ii). We can carry out the construction so that (a) $R^{+}$and $R^{-}$are to the left and to the right of the $y$-axis, respectively, (b) $r \in R^{-}$, and (c) $m_{0}$ is oriented from $r$ away from the other contact point(s), and the positive $y$-direction lies counterclockwise to it. See Fig. 3. This still leaves us with some freedom in fixing the coordinate frame. We later impose further constraints on it to facilitate certain steps in our analysis.

The presence of $q$ adds an extra level of complication to the proof. We note that in the configuration shown in Fig. 1, choosing $p_{0}$ to be any vertex of the hull, say, the lowest point, the weighted set $R$ has a central bichromatic point $q$, as shown in Fig. 31(ii). As will follow from our analysis, the bounds in both Theorems 1.1 and 1.3 improve to $2 n-2$, for $n$ odd, when $q$ does not exist.

Let $P^{+}$(resp., $P^{-}$) denote the set of points of $P \backslash\left\{p_{0}\right\}$ that project from $p_{0}$ to points of $R^{+}$(resp., $R^{-}$). Points projecting to $q$ are split between $P^{+}$and $P^{-}$. This can be best 
visualized by a plane $\pi_{0}$ that separates $P^{+}$and $P^{-}$. If $q$ does not exist, $\pi_{0}$ is the plane spanned by $p_{0}$ and the $y$-axis in $\pi$. If $q$ exists, $\pi_{0}$ cuts the line containing the preimages of $q$ into two pieces, one of which contains $w\left(q^{+}\right)$preimages and the other contains $w\left(q^{-}\right)$preimages. Without loss of generality, we may assume that the $w\left(q^{+}\right)$preimages closest to $p_{0}$ belong to $P^{+}$.

A Brief Overview of the Proof of Theorem 1.3. In Section 4 we construct a set $F$ of mutually non-convergent segments in $\pi$, whose endpoints belong to $R$. With few exceptions, the segments in $F$ connect points of $R^{+}$to points of $R^{-}$. Observe that a segment in $\pi$ connecting two points $r_{1}$ and $r_{2}$ of $R$ is in fact a projection to $\pi$ (through $p_{0}$ ) of a segment connecting two points of $P$ which project to $r_{1}$ and $r_{2}$, respectively. Moreover, if $e^{\prime}$ and $f^{\prime}$ are two segments in $\mathbb{R}^{3}$ whose respective projections onto $\pi$ are $e$ and $f$, and if $e$ and $f$ are non-convergent, then so are $e^{\prime}$ and $f^{\prime}$, as is easily checked.

This is more or less the strategy used in our preceding study [14]. In the setup assumed there, each point of $R$ was the image of a unique point of $P \backslash\left\{p_{0}\right\}$, and the number of segments in $F$ was roughly $|R|$. Then the segments in 3-space that project to the segments of $F$, together with the segments connecting $p_{0}$ to each of the other points of $P$, yielded the desired set of mutually non-convergent segments determined by $P$.

However, the crucial difference between the setup in Theorem 1.3 and that in the main theorem of [14] is that now the central projection from $p_{0}$ of $P$ onto $R$ may be many-to-one, because $P$ may have many points that are collinear with $p_{0}$, in which case they all project to a single point of $R$. As a result, the set $R$ may consist of many fewer than $|P|-1$ points, and hence the set $F$ may not contain the desired number of mutually non-convergent segments. (In the extreme case, $R$ might consist of only two points and $F$ of just one segment!)

To resolve this issue, we take advantage of the fact that many points in $P$ project to the same point in $\pi$, and use it to map each segment $f \in F$ to a set $E(f)$ of pairwise non-convergent segments in 3-space, determined by $P$, and lying in the plane $h$ spanned by $p_{0}$ and $f$. The sum of the sizes $|E(f)|$, over $f \in F$, is at least equal to the desired bound on the number of different directions.

In more detail, let $f \in F$ be one of the segments we collected on $\pi$. Let $\ell$ be the line in $\pi$ that contains $f$, and let $h$ be the plane spanned by $\ell$ and $p_{0}$. Put $P_{h}=P \cap h$, and define $P_{h}^{+}=P^{+} \cap h$ and $P_{h}^{-}=P^{-} \cap h$. Note that any segment that connects two points in $P_{h}$ projects to a segment in $\pi$ that is collinear with $f$, and thus $f$ is the only segment in $F$ that can be obtained in such a way. To compensate for this "waste", we apply Theorem 3.1 (stated and proved in Section 3), which implies the existence of a (sufficiently large) set $E(f)$ of pairwise non-convergent segments in $P_{h}$. Each segment $e \in E(f)$ either connects a point of $P_{h}^{+}$to a point of $P_{h}^{-}$, or connects $p_{0}$ to some point in $P_{h}$, such that the projection $e^{*}$ of $e$ from $p_{0}$ on the line $\ell$ supporting $f$ either fully contains $f$, or is a point, outside the interior of $f$; see Fig. 4(a).

We note that the proof of Theorem 3.1 itself, which is a far-reaching bipartite variant of Ungar's theorem, is rather intricate, and occupies a significant portion of the paper. Although the proof bears some "syntactic" similarities to the proof of our main Theorem 1.3, it deals with a completely different scenario, and is therefore presented separately, as a stand-alone result (which we believe to be of independent interest). 


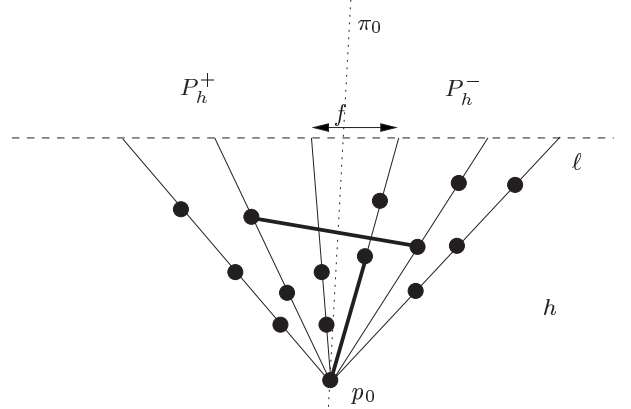

(a)

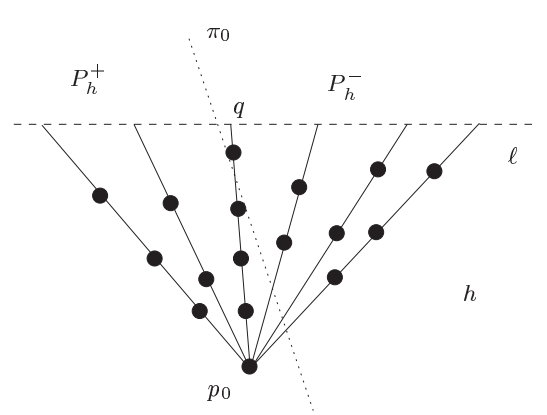

(b)

Fig. 4. The sets $P_{h}^{+}$and $P_{h}^{-}$. (a) The case where the central bichromatic point $q$ (if it exists at all) does not lie on $\ell$. (b) The case where $q$ exists and lies on $\ell$.

Finally, we let $E$ denote the union of all the sets $E(f)$. Using a fairly intricate analysis, based on the properties of the construction in Theorem 3.1 noted above, we show (assuming that $n$ is odd) that (a) $E$ consists of at least $2 n-5$ segments (Section 4.4), and (b) every pair of distinct segments in $E$ are non-convergent and therefore non-parallel (Section 4.5). Once (a) and (b) are established, Theorems 1.3 and 1.1 follow, because the directions of the segments in $E$ are all different.

We emphasize once again that Theorem 1.3 is considerably stronger than Theorem 1.1. Besides being of independent interest, we expect this strengthening to be useful for extending our results to higher dimensions, using induction on the dimension; see the concluding section for more details.

\section{A Bipartite Ungar-Type Theorem}

A crucial ingredient of our analysis is the following variant of Ungar's theorem, which we believe to be of independent interest.

Theorem 3.1. Let $X^{+}$and $X^{-}$be two finite sets of points in the plane, and let $p_{0}$ be a point in the plane, such that $p_{0}$ is an extreme point of $X^{+} \cup X^{-} \cup\left\{p_{0}\right\}$, and there is a line through $p_{0}$ that strictly separates $X^{+}$and $X^{-}$. We also assume that $0<\left|X^{-}\right| \leq\left|X^{+}\right|$ and that the innermost ray from $p_{0}$ to a point of $X^{+}$(forming the smallest angle with the separating line) contains more than $\left|X^{+}\right|-\left|X^{-}\right|$points. Then one can select at least $\left|X^{+}\right|+\left|X^{-}\right|+1$ pairwise non-convergent and non-collinear segments connecting points of $X^{+} \cup\left\{p_{0}\right\}$ to points of $X^{-} \cup\left\{p_{0}\right\}$.

We remark that the " +1 " term in the above bound is crucial for our analysis, and that we may lose this term if the assumption on the points in the innermost ray does not hold, as is illustrated in Fig. 5, where $\left|X^{+}\right|+\left|X^{-}\right|+1=5$ but at most four pairwise non-convergent segments can be selected.

Corollary 3.2. Assume the conditions of Theorem 3.1, with the difference that the innermost ray from $p_{0}$ to a point of $X^{+}$contains exactly $\left|X^{+}\right|-\left|X^{-}\right|$points. Then one 


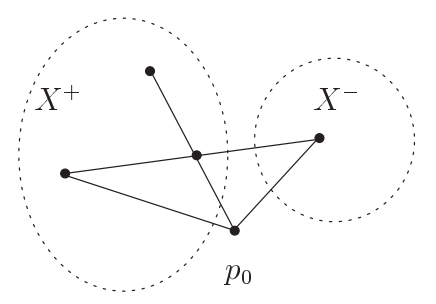

Fig. 5. An example where Theorem 3.1 does not hold.

can select at least $\left|X^{+}\right|+\left|X^{-}\right|$pairwise non-convergent and non-collinear segments connecting points of $X^{+} \cup\left\{p_{0}\right\}$ to points of $X^{-} \cup\left\{p_{0}\right\}$.

We note that Ungar's theorem "almost" follows from Theorem 3.1 and its corollary. That is, let $P$ be a set of $n$ non-collinear points in the plane, where $n$ is even. Pick an extreme point $p_{0}$ of $P$, and find a line that passes through $p_{0}$ and splits $P \backslash\left\{p_{0}\right\}$ into two subsets $X^{+}, X^{-}$whose sizes are as equal as possible. Suppose that $\left|X^{+}\right| \geq\left|X^{-}\right|$. Then the innermost ray from $p_{0}$ to points of $X^{+}$must contain at least $\left|X^{+}\right|-\left|X^{-}\right|$points, for otherwise we could have transferred these points to $X^{-}$and get a split with a smaller size difference. If the number of points on the innermost ray is larger than $\left|X^{+}\right|-\left|X^{-}\right|$, then Theorem 3.1 applies, and yields at least $\left|X^{+}\right|-\left|X^{-}\right|+1=|P|$ pairwise non-convergent segments connecting the points of $P$, which implies (and is much stronger than) Ungar's theorem. However, if the number of points on the innermost ray is equal to $\left|X^{+}\right|-\left|X^{-}\right|$, then only Corollary 3.2 can be applied, and it only yields $\left|X^{+}\right|-\left|X^{-}\right|=|P|-1$ pairwise non-convergent segments connecting the points of $P$, one shorter than Ungar's theorem gives. We leave it as an open problem to determine whether Ungar's theorem can always be deduced from Theorem 3.1 and Corollary 3.2.

Proof of Corollary 3.2. Remove one point from $X^{+}$which is not on the innermost ray from $p_{0}$ to a point of $X^{+}$(note that $X^{+}$is not fully contained in that ray, since $\left|X^{-}\right|>0$ ), and apply Theorem 3.1 to the resulting set of points.

Proof of Theorem 3.1. Fix an $(x, y)$-coordinate system in the plane. We apply a standard duality transform that maps a point $p=\left(p_{1}, p_{2}\right)$ to the line $p^{*}$ with the equation $y+p_{1} x+p_{2}=0$. Vice versa, a non-vertical line $l$ with the equation $y+l_{1} x+l_{2}=0$ is mapped to the point $l^{*}=\left(l_{1}, l_{2}\right)$. Consequently, any two parallel lines are mapped into points having the same $x$-coordinate. It is often convenient to imagine that the dual picture lies in another, so-called dual, plane, different from the original one, which is referred to as the primal plane.

The above mapping is incidence and order preserving, in the sense that $p$ lies above, on, or below $\ell$ if and only $\ell^{*}$ lies above, on, or below $p^{*}$, respectively. The points of a non-vertical segment $e=a b$ in the primal plane are mapped to the set of all lines in the closed double wedge $e^{*}$, which is bounded by $a^{*}$ and $b^{*}$ and does not contain the vertical direction. All of these lines pass through the point $q=a^{*} \cap b^{*}$, which is called the apex of the double wedge $e^{*}$. All double wedges used in this paper are assumed to be closed, and they never contain the vertical direction. 

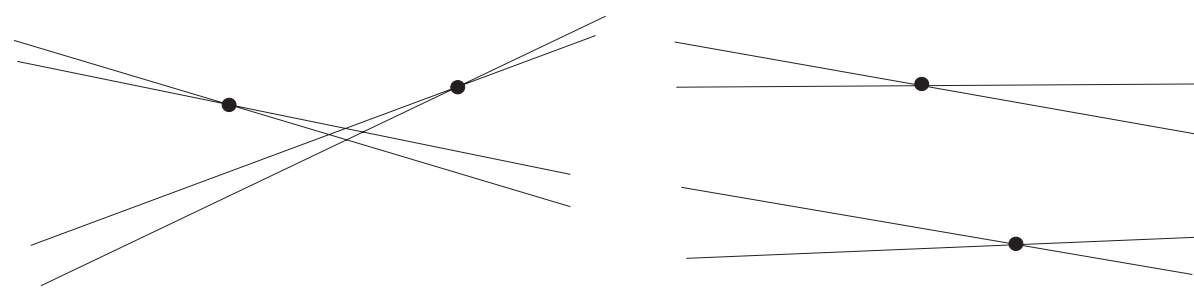

Fig. 6. Two possible kinds of convergent double wedges.

We call two double wedges convergent if their apices are distinct and the apex of neither of them is contained in the other. See Fig. 6.

It is easy to see that, according to this definition, two non-collinear segments in the primal plane are convergent if and only if they are mapped to convergent double wedges.

Without loss of generality, we assume that $p_{0}$ is the origin, that $X^{+}$lies to the left of the $y$-axis, that $X^{-}$lies to its right, and that both sets lie below the $x$-axis; see Fig. 7(a). The duality maps $p_{0}$ into the $x$-axis, which we denote as $\ell_{0}$, the lines connecting $p_{0}$ to points in $X^{+}$(resp., $X^{-}$) to points on the negative (resp., positive) $x$-axis, and the points of $X^{+}$(resp., $X^{-}$) to lines with positive (resp., negative) slopes; see Fig. 7(b). Let $\Gamma^{+}, \Gamma^{-}$denote the set of lines dual to the points of $X^{+}, X^{-}$, respectively. Enumerate the points dual to the lines connecting $p_{0}$ to the points of $X^{+}$as $q_{1}, \ldots, q_{k}$ in this leftto-right order, and the points dual to the lines connecting $p_{0}$ to the points of $X^{-}$as $q_{1}^{\prime}, \ldots, q_{\ell}^{\prime}$ in this right-to-left order; thus $q_{1}$ is the leftmost point and $q_{1}^{\prime}$ is the rightmost. Put $n^{+}=\left|\Gamma^{+}\right|=\left|X^{+}\right|, n^{-}=\left|\Gamma^{-}\right|=\left|X^{-}\right|$.

Define $\Lambda^{-}:=\Gamma^{-} \cup\left\{\ell_{0}\right\}$. Let $\Lambda^{e}$ denote the set of $n^{+}-n^{-}$lines of $\Gamma^{+}$that pass through $q_{1}$ and have the shallowest (smallest in absolute value) slopes; we refer to these lines as excess lines. Define $\Lambda^{+}=\Gamma^{+} \backslash \Lambda^{e}$. We have $\left|\Lambda^{-}\right|=\left|\Lambda^{+}\right|+1=n^{-}+1$. We note that by an appropriate choice of the coordinate frame in the duality transform, we may assume that the slopes of the excess lines are the smallest among all lines in $\Gamma^{+}$.

Constructing junctions. We apply an iterative pruning process that constructs a sequence of vertices ("junctions") $v_{1}, \ldots, v_{m}$ which are intersection points of lines from $\Lambda^{-}$ and lines from $\Lambda^{+}$, and sets of intermediate vertices ("stations") between successive

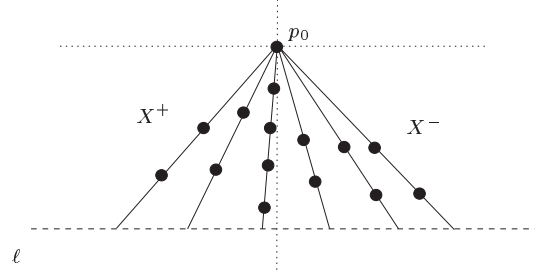

(a)

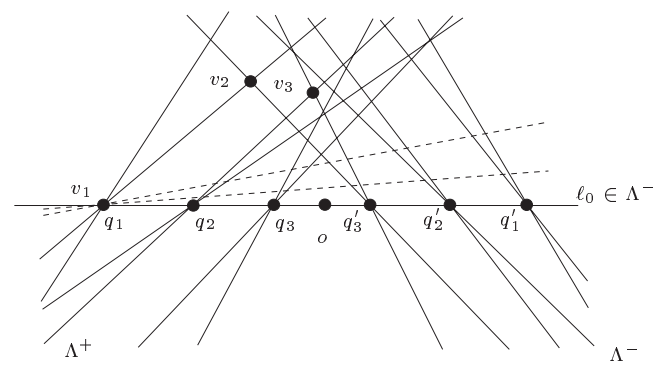

(b)

Fig. 7. The setup in Theorem 3.1. (a) The primal configuration. (b) The dual configuration. Since $\left|X^{+}\right|=8$ and $\left|X^{-}\right|=6$, we have two excess lines, shown dashed. 


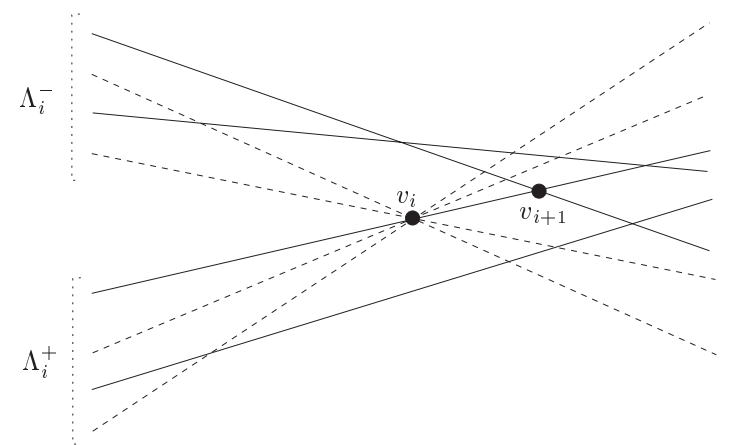

Fig. 8. Constructing the junction $v_{i}$ in $J$. The dashed lines, two from $\Lambda^{+}$and two from $\Lambda^{-}$, are removed at $v_{i}$. The next junction $v_{i+1}$ is also shown.

junctions, as well as a set of "termini" to the right of the rightmost junction. The sequence $J$ of junctions $\left\langle v_{1}, v_{2}, \ldots, v_{k}\right\rangle$ is constructed as follows.

STEP 1 . Set $i:=1$ and $\Lambda_{1}^{+}:=\Lambda^{+}, \Lambda_{1}^{-}:=\Lambda^{-}$.

STEP 2. If $\Lambda_{i}^{+}=\emptyset$, the construction of $J$ terminates. Otherwise, as guaranteed by the construction, neither set is empty. Let $v_{i}$ be the leftmost intersection point between a line in $\Lambda_{i}^{+}$and a line in $\Lambda_{i}^{-}$. Let $d_{i}^{+}$(and $d_{i}^{-}$) denote the number of lines of $\Lambda_{i}^{+}$(and $\Lambda_{i}^{-}$, respectively) incident to $v_{i}$, and put $d_{i}:=\min \left\{d_{i}^{+}, d_{i}^{-}\right\}$. Define $\Lambda_{i+1}^{+}\left(\right.$resp., $\left.\Lambda_{i+1}^{-}\right)$as the set of lines obtained from $\Lambda_{i}^{+}$(resp., $\Lambda_{i}^{-}$) by deleting from it the $d_{i}$ lines that are incident to $v_{i}$ and have the largest (resp., smallest) slopes among those incident lines. (That is, if $d_{i}^{+}=d_{i}^{-}$, then all lines incident to $v_{i}$ are deleted; otherwise, if, say, $d_{i}^{+}>d_{i}^{-}$, we are left with $d_{i}^{+}-d_{i}^{-}$lines through $v_{i}$ that belong to $\Lambda_{i}^{+}$and separate the deleted elements of $\Lambda_{i}^{+}$from the deleted elements of $\Lambda_{i}^{-}$. See Fig. 8, where $d_{i}=d_{i}^{-}=2, d_{i}^{+}=3$, and the dashed lines, two from $\Lambda_{i}^{+}$and two from $\Lambda_{i}^{-}$, are removed at $v_{i}$.) Set $i:=i+1$, and repeat Step 2.

Note that, due to the special structure of the arrangement, we have $v_{1}=q_{1}$ and $d_{1}=1$. See Fig. 7(b). Recall also that the excess lines do not participate in the junction construction process.

It is easy to verify the following properties of this construction.

\section{Claim 3.3.}

(i) $\left|\Lambda_{i}^{-}\right|=\left|\Lambda_{i}^{+}\right|+1$, for each $i=1, \ldots, k ; \Lambda_{k+1}^{+}=\emptyset$ and $\left|\Lambda_{k+1}^{-}\right|=1$.

(ii) For every $1 \leq i<j \leq k$, the junction $v_{i}$ lies in the left unbounded face $f_{j}$ of $\mathcal{A}\left(\Lambda_{j}^{+} \cup \Lambda_{j}^{-}\right)$which separates $\Lambda_{j}^{+}$and $\Lambda_{j}^{-}$at $x=-\infty$ (and whose rightmost vertex is $\left.v_{j}\right)$. $v_{i}$ lies in the interior of $f_{j}$ if $d_{i}^{+}=d_{i}^{-}$; otherwise it may lie on the boundary of $f_{j}$.

(iii) $\sum_{i=1}^{k} d_{i}=\left|\Lambda^{+}\right|=n^{-}$.

Collecting stations. Next, between any two consecutive junctions $v_{i}$ and $v_{i+1}$, for $1 \leq$ $i<k$, we specify $d_{i}+d_{i+1}-1$ further vertices of $\mathcal{A}\left(\Lambda^{+} \cup \Lambda^{-}\right)$, called stations (thus, the excess lines are still kept out of the construction). 


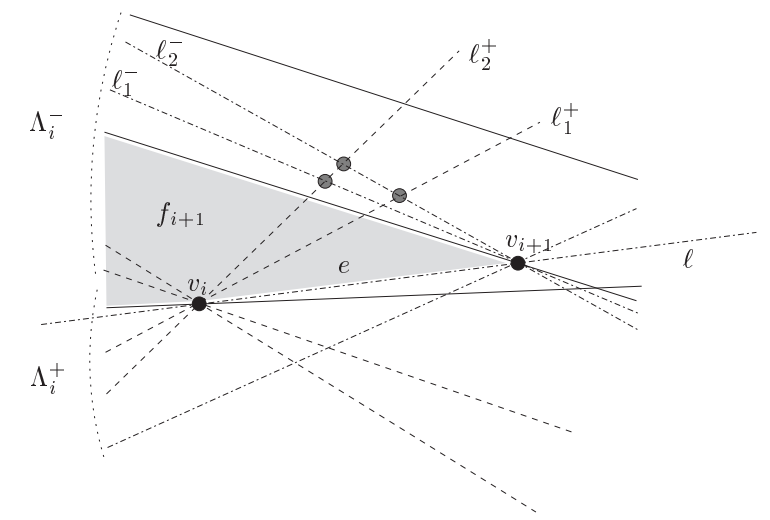

Fig. 9. Collecting stations between $v_{i}$ and $v_{i+1}$. We have $d_{i}=d_{i+1}=2$. The lines removed at $v_{i}$ are drawn as dashed, and those removed at $v_{i+1}$ are drawn as dashed-dotted.

Fix an index $1 \leq i<k$, and consider the vertical slab between $v_{i}$ and $v_{i+1}$. By Claim 3.3(ii), $v_{i}$ lies inside or on the boundary of the face $f_{i+1}$ of $\mathcal{A}\left(\Lambda_{i+1}^{+} \cup \Lambda_{i+1}^{-}\right)$, whose rightmost vertex is $v_{i+1}$. See Fig. 9. Hence, the segment $e=v_{i} v_{i+1}$ is contained in the closure of $f_{i+1}$. Now at least one of the following two conditions is satisfied: (a) all the $d_{i}$ lines removed from $\Lambda_{i}^{+}$and all the $d_{i+1}$ lines removed from $\Lambda_{i+1}^{-}$pass strictly above $e$ (except possibly for its endpoints), or (b) all the $d_{i}$ lines removed from $\Lambda_{i}^{-}$and all the $d_{i+1}$ lines removed from $\Lambda_{i+1}^{+}$pass strictly below $e$.

Indeed, if $v_{i}$ lies in the interior of $f_{i+1}$ then the $d_{i+1}$ lines of $\Lambda_{i+1}^{+}$(resp., of $\Lambda_{i+1}^{-}$) that are removed at $v_{i+1}$ pass strictly below (resp., above) $v_{i}$. In this case the validity of either (a) or (b) follows by considering the position of $v_{i}$ among the lines of $\Lambda_{i}^{+} \cup \Lambda_{i}^{-}$ that are removed at $v_{i}$. If $v_{i}$ lies on the boundary of $f_{i+1}$ (as shown in Fig. 9), then it has to lie on a line of $\Lambda_{i+1}^{+} \cup \Lambda_{i+1}^{-}$, say it lies on a line $\ell$ of $\Lambda_{i+1}^{+}$(as shown in the figure). Then all the $d_{i+1}$ lines removed from $\Lambda_{i+1}^{-}$pass strictly above $v_{i}$ and $e$. Now the line $\ell$ belongs to $\Lambda_{i}^{+}$and passes through $v_{i}$. Since it was not removed at $v_{i}$, all the $d_{i}$ lines of $\Lambda_{i}^{+}$that were removed pass strictly above $e$, by construction, so (a) holds. If $v_{i}$ lies on a line of $\Lambda_{i+1}^{-}$, a symmetric argument shows that (b) holds.

Assume, by symmetry, that (a) holds. Denote the lines removed from $\Lambda_{i}^{+}$by $\ell_{1}^{+}, \ldots$, $\ell_{d_{i}}^{+}$, listed according to increasing slopes, and those removed from $\Lambda_{i+1}^{-}$by $\ell_{1}^{-}, \ldots, \ell_{d_{i+1}}^{-}$, listed according to decreasing slopes. See Fig. 9. Define the set of stations $S_{i}$ in the vertical slab between $v_{i}$ and $v_{i+1}$ as the collection of all intersection points of $\ell_{d_{i}}^{+}$with the lines $\ell_{1}^{-}, \ldots, \ell_{d_{i+1}}^{-}$, and all intersection points of $\ell_{d_{i+1}}^{-}$with the lines $\ell_{1}^{+}, \ldots, \ell_{d_{i}}^{+}$. Clearly, we have $\left|S_{i}\right|=d_{i}+d_{i+1}-1$ such points; see Fig. 9. We refer to the grid-like crossing pattern between the lines $\ell_{1}^{+}, \ldots, \ell_{d_{i}}^{+}$and the lines $\ell_{1}^{-}, \ldots, \ell_{d_{i+1}}^{-}$, as the upper grid between $v_{i}$ and $v_{i+1}$. The collected stations lie on the "upper rim" of that grid. In complete analogy, when case (b) applies, we collect stations along the "lower rim" of the lower grid between $v_{i}$ and $v_{i+1}$.

The description so far matches the one given in [14]. We now describe the new features of the present collection process. They involve (a) collecting "excess stations" for the excess lines, and (b) collecting vertices (that we refer to as "termini") to the right of $v_{k}$. 
Collecting excess stations. The collection of excess stations proceeds as follows. As we collect the junctions $v_{i}$, we maintain a subset $\Lambda_{i}^{e}$ of "surviving" excess lines. For each $i$, the lines in $\Lambda_{i}^{e}$ satisfy the property that they pass below or through each of the junctions $v_{1}, \ldots, v_{i}$. Initially, $\Lambda_{1}^{e}=\Lambda^{e}$, all of whose lines clearly satisfy this property (they pass through $v_{1}$ ). When we reach a new junction $v_{j}$, we remove certain lines from $\Lambda_{j-1}^{e}$. When an excess line is removed, we associate with it a new excess station that lies somewhere to the left of $v_{j}$. Typically, but not always, it will be a grid vertex between $v_{j-1}$ and $v_{j}$. To disambiguate between the two kinds of stations, we sometimes refer to the previously constructed stations as standard stations. The removal of excess lines and the construction of excess stations proceed according to the following rule:

(i) Our default option is to use the upper grid for collecting intermediate (standard) stations between $v_{j-1}$ and $v_{j}$. Recall that, for this to be possible, all lines of $\Lambda^{-}$incident to $v_{j}$ and removed there have to pass strictly above $v_{j-1}$, and all lines of $\Lambda^{+}$incident to $v_{j-1}$ and removed there have to pass strictly above $v_{j}$. If the first condition is violated then the shallowest line of $\Lambda^{-}$incident to $v_{j}$ and removed there also passes through $v_{j-1}$ (by Claim 3.3(ii), it cannot pass below $v_{j-1}$ ), and if the second condition is violated then the shallowest line of $\Lambda^{+}$incident to $v_{j-1}$ and removed there passes through or below $v_{j}$. Thus, if none of the two latter conditions arise, we use the upper grid.

Assuming this to be the case, we remove each surviving excess line that passes above $v_{j}$. The removed excess lines meet the steepest line of $\Lambda^{-}$incident to $v_{j}$ at points that lie along the upper grid and are further to the right of all the other grid points (and thus to the right of all the standard stations in $S_{j-1}$ ). This latter property is a consequence of the fact that all these excess lines pass below or through $v_{j-1}$ and have slopes smaller than those of the lines of $\Lambda^{+}$that are incident to $v_{j-1}$; see Fig. 10 .

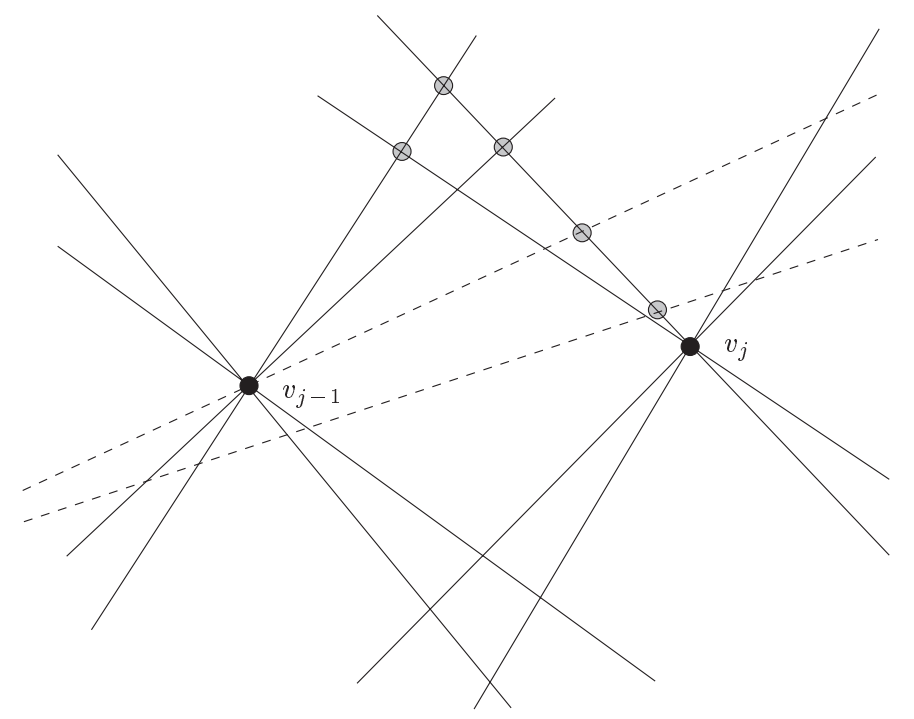

Fig. 10. Charging excess lines of $\Lambda_{j-1}^{e}$ that pass above $v_{j}$ to excess stations in the upper grid between $v_{j-1}$ and $v_{j}$. 


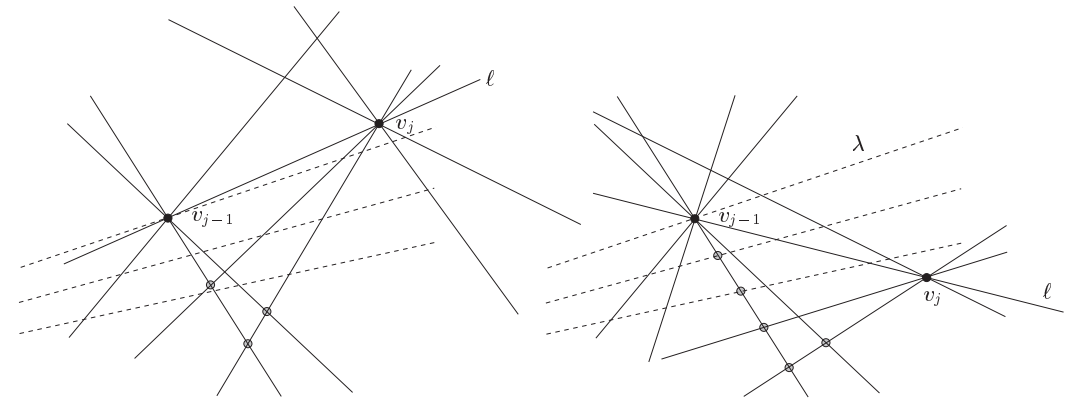

(a)

(b)

Fig. 11. Using the lower grid between $v_{j-1}$ and $v_{j}$.

In conclusion, each removed excess line is associated with a new upper grid vertex of the arrangement, and these are the excess stations that we have promised to collect. We set $\Lambda_{j}^{e}$ to be the set of surviving excess lines, which still pass through or below $v_{j}$ (so the invariant continues to hold), and continue the process with $j:=j+1$.

(ii) Suppose that we have to use the lower grid for collecting intermediate stations between $v_{j-1}$ and $v_{j}$. As just mentioned, the lower grid has to be used either (a) when $v_{j}$ lies on or above at least one of the $d_{j-1}$ lines of $\Lambda^{+}$incident to $v_{j-1}$ and removed there, or (b) when $v_{j-1}$ lies on the shallowest of the $d_{j}$ lines of $\Lambda^{-}$incident to $v_{j}$ and removed there.

In case (a), let $\ell$ denote the shallowest line in $\Lambda^{+}$through $v_{j-1}$ that is removed at $v_{j-1}$. Refer to Fig. 11(a), and note that $v_{j}$ lies on or above $\ell$. In this case each excess line in $\Lambda_{j-1}^{e}$ must pass below $v_{j}$, because it passes below or through $v_{j-1}$ and its slope is smaller than that of $\ell$. Hence, in this case we do not remove any excess line, and thus set $\Lambda_{j}^{e}:=\Lambda_{j-1}^{e}$. In particular, the invariant property holds for $\Lambda_{j}^{e}$ in this case, and we continue the collection process with $j:=j+1$.

In case (b), which is depicted in Fig. 11(b), let $\ell$ denote the shallowest line in $\Lambda^{-}$ through $v_{j}$ that is removed at $v_{j}$. $\ell$ passes also through $v_{j-1}$. We use the lower grid to construct excess stations for the excess lines of $\Lambda_{j-1}^{e}$ that pass above $v_{j}$. These will be the intersection points of these lines with the steepest line of $\Lambda^{-}$incident to $v_{j-1}$. Because of the slope conditions, these points lie to the left of all the standard stations between $v_{j-1}$ and $v_{j}$. However, if there exists an excess line $\lambda$ through $v_{j-1}$, this procedure will fail to produce an additional excess station for $\lambda$. To gain such a station elsewhere, we observe that $j-1 \neq 1$ ( since $\ell_{0}$ is the only line of $\Lambda^{-}$through $v_{1}$, so that it is deleted there and does not belong to $\Lambda_{2}^{-}$), and that we must have used the upper grid between $v_{j-2}$ and $v_{j-1}$. This holds because $v_{j-2}$ must lie on or below $\ell$ and on or above $\lambda$. Hence, all the lines of $\Lambda^{+}$incident to $v_{j-2}$ must pass strictly above $v_{j-1}$ (since they have slopes larger than that of $\lambda$ ), and all the lines of $\Lambda^{-}$incident to $v_{j-1}$ and removed there must pass strictly above $v_{j-2}$ (since they are all steeper than $\ell$ ). Note that the number of lines of $\Lambda^{-}$ through $v_{j-1}$ is greater than $d_{j-1}$, because this set also contains $\ell$, which has not been removed at $v_{j-1}$. Using that extra line, we can therefore gain one additional intersection point as the required excess station in the upper grid between $v_{j-2}$ and $v_{j-1}$. 


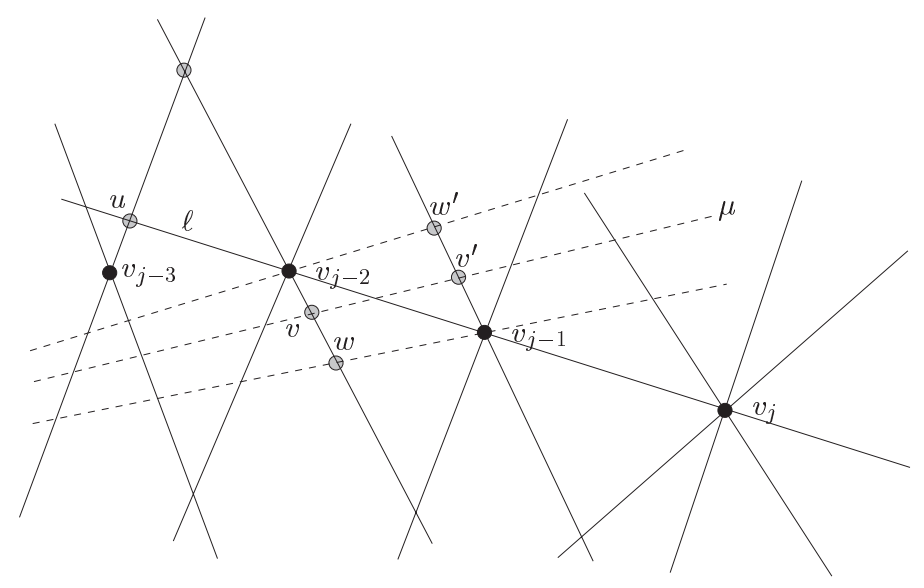

Fig. 12. Handling the modified case (b), where $\ell$ passes through additional vertices preceding $v_{j-1}$.

However, one such extra grid station may fail to exist if $v_{j-2}$ also lies on $\ell$. Refer to Fig. 12.

In this case it is easily seen that, as far as the collection of standard stations goes, we can use the lower grid between $v_{j-2}$ and $v_{j-1}$ instead of the upper grid. Indeed, all the lines of $\Lambda^{-}$incident to $v_{j-2}$ and removed there pass below $v_{j-1}$ (because $\ell$ passes through $v_{j-1}$ and is not removed there), and all the lines of $\Lambda^{+}$incident to $v_{j-1}$ and removed there pass below $v_{j-2}$ (because $\lambda$ passes through $v_{j-1}$ and below $v_{j-2}$ ). If $v_{j-2}$ is not incident to an excess line, then all excess lines in $\Lambda_{j-2}^{e}$ that pass through or above $v_{j-1}$ (including $\lambda$ ) determine excess stations on the lower grid between $v_{j-2}$ and $v_{j-1}$. Hence in this case we obtain on the lower grid one additional excess station, formed by $\lambda$, and can therefore quit this process. If $v_{j-2}$ is incident to an excess line, we attempt to collect an extra excess station in the upper grid between $v_{j-3}$ and $v_{j-2}$, exploiting, as above, the excess of lines of $\Lambda^{-}$at $v_{j-2}$. Again, this may fail if $v_{j-3}$ also lies on $\ell$, so we move to the lower grid between $v_{j-3}$ and $v_{j-2}$, and we keep applying this backtracking process until we reach a junction $v_{s}$ that lies strictly below $\ell$. This will happen, if not earlier, when we reach $v_{1}$, since the only line of $\Lambda^{-}$incident to $v_{1}$ is $\ell_{0}$, which is different from $\ell$.

To recap, this process creates an excess station for each excess line removed at $v_{j}$. Note that if the construction had to backtrack from $v_{j}$ through several preceding junctions, then $\ell$ is the shallowest line of $\Lambda_{j}^{-}$that passes through $v_{j}$. Hence, if backtracking will also be required at some later junction $v_{j^{\prime}}$, for $j^{\prime}>j$, then the process will have to terminate at a junction to the right of $v_{j}$ (because no surviving line of $\Lambda_{j}^{-}$passes through $\left.v_{j}\right)$. That is, the backtracking processes are independent of each other, and none of them affects any of the preceding ones.

Collecting termini. Finally, consider the last junction $v_{k}$ and the final set $\Lambda_{k}^{e}$ of surviving excess lines. There are $d_{k}$ lines of $\Lambda^{-}$as well as $d_{k}$ lines of $\Lambda^{+}$that pass through $v_{k}$, and there is another surviving line $\ell$ of $\Lambda^{-}$, which passes through or above $v_{k}$. Our goal is to collect $d_{k}+\left|\Lambda_{k}^{e}\right|+1$ additional vertices of $\mathcal{A}(\Lambda)$ to the right of $v_{k}$, to which we refer as termini. 


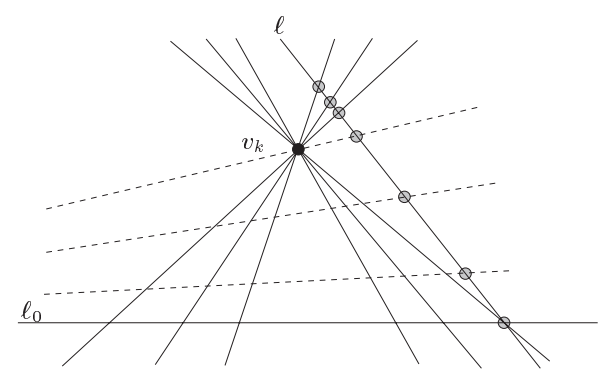

(a)

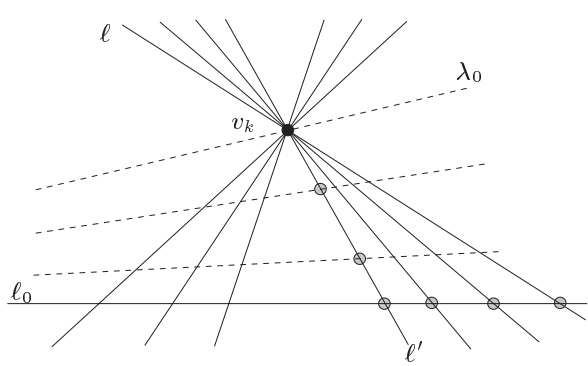

(b)

Fig. 13. Collecting termini.

If $\ell$ passes above $v_{k}$, then we obtain on it the distinct intersection points with $\ell_{0}$, with the excess lines in $\Lambda_{k}^{e}$, and with the $d_{k}$ lines of $\Lambda^{+}$through $v_{k}$ (it is easy to verify that all these intersection points are indeed distinct); see Fig. 13(a). Altogether, we collect $d_{k}+\left|\Lambda_{k}^{e}\right|+1$ termini.

If $\ell$ passes through $v_{k}$ (see Fig. 13(b)), let $\ell^{\prime}$ denote the steepest line of $\Lambda^{-}$incident to $v_{k}$. We charge each of the $d_{k}+1$ lines of $\Lambda^{-}$incident to $v_{k}$ to its intersection with $\ell_{0}$. In addition, each excess line in $\Lambda_{k}^{e}$, with the exception of the excess line $\lambda_{0}$ that passes through $v_{k}$ (if there is such a line), meets $\ell^{\prime}$ at a vertex, and we add these vertices to the set of collected termini; their $x$-coordinates are all distinct, and lie to the right of $v_{k}$ and to the left of any point $q_{j}^{\prime}$ charged by the lines of $\Lambda^{-}$incident to $v_{k}$. Altogether we collect at least $d_{k}+\left|\Lambda_{k}^{e}\right|$ termini. The only case in which we do not obtain $d_{k}+\left|\Lambda_{k}^{e}\right|+1$ termini is when there is an excess line $\lambda_{0}$ through $v_{k}$. In this case we must have used the upper grid between $v_{k-1}$ and $v_{k}$, which is argued as in case (ii) of the preceding analysis. As above, we can gain an extra excess station in this upper grid, because the number of lines of $\Lambda^{-}$through $v_{k}$ is in fact at least $d_{k}+1$. Again, the same technical difficulty that we faced earlier may arise here as well, when $v_{k-1}$ also lies on $\ell$. We resolve this exactly as before, backtracking to the left through junctions $v_{j}$ that lie on $\ell$, switch to lower grids between them without decreasing the number of collected stations, and gaining the desired extra station when we reach a junction $v_{j}$ that lies strictly below $\ell$ or that is not incident to an excess line.

In both cases we have managed to charge an extra terminus for every excess line left in $\Lambda_{k}^{e}$, and an additional terminus for the extra surviving line $\ell$ of $\Lambda^{-}$. Note that all termini, or all but one, lie to the right of $v_{k}$.

Adding these termini to the junctions and stations, we obtain, excluding the excess stations and termini, and recalling that $d_{1}=1$, a total of

$$
\begin{gathered}
\left(d_{1}+d_{2}-1\right)+\left(d_{2}+d_{3}-1\right)+\cdots+\left(d_{k-1}+d_{k}-1\right)+k+\left(d_{k}+1\right) \\
=1+2 \sum_{i=1}^{k} d_{i}=2 n^{-}+1
\end{gathered}
$$

vertices. Hence, since we manage to collect one additional vertex for each excess line, we obtain a total of $2 n^{-}+1+\left(n^{+}-n^{-}\right)=n^{+}+n^{-}+1$ vertices. Observe that all the collected vertices are either on $\ell_{0}$ or are intersection points of lines of (the original) $\Lambda^{+}$ 
with lines of (the original) $\Lambda^{-}$. In other words, each of the collected vertices represents a segment in the primal plane, connecting a point of $X^{+} \cup\left\{p_{0}\right\}$ to a point of $X^{-} \cup\left\{p_{0}\right\}$.

Let $Q$ denote the set of all collected junctions, stations, and termini. Associate with each element $q \in Q$ the maximal double wedge $W(q)$ (not containing the vertical line through $q$ ), which is bounded by a pair of lines passing through $q$.

To complete the proof of the theorem, we show that the collected wedges are pairwise non-convergent.

Claim 3.4. The set $\{W(q) \mid q \in Q\}$ of $n$ double wedges has no two convergent elements.

Proof. Let $u, v \in Q$ with $u$ lying to the left of $v$. Recalling the definition of convergent double wedges, we need to show that either $u \in W(v)$ or $v \in W(u)$. We distinguish between several cases:

Case A: Both $u$ and $v$ are junctions. Put $u=v_{i}$ and $v=v_{j}$, with $i<j$. Then $W(v)$ is bounded by a line $\ell \in \Lambda_{j}^{+}$and by a line $\ell^{\prime} \in \Lambda_{j}^{-}$. By Claim 3.3(ii), $v_{i}$ lies between these two lines, and thus belongs to $W(v)$.

Case B: $u$ is a junction and $v$ is a (standard or excess) station to the left of $v_{k}$. Put $u=v_{i}$ and let $S_{j}$ be the set of stations that contains $v$, where $i \leq j<k$. Then $W(v)$ is bounded by two lines $\ell, \ell^{\prime}$, where either $\ell \in \Lambda_{j}^{+} \cup \Lambda_{j}^{e}$ and $\ell^{\prime} \in \Lambda_{j+1}^{-}$(if $v$ lies on the upper grid), or $\ell \in \Lambda_{j+1}^{+} \cup \Lambda_{j}^{e}$ and $\ell^{\prime} \in \Lambda_{j}^{-}$(if $v$ lies on the lower grid). By construction, we have, in both cases, $\ell \in \Lambda_{j}^{+} \cup \Lambda_{j}^{e}$ and $\ell^{\prime} \in \Lambda_{j}^{-}$. If $\ell \in \Lambda_{j}^{+}$, the analysis is completed as in Case A. If $\ell \in \Lambda_{j}^{e}$, it passes through or below $v_{i}$, so the same analysis applies here as well.

Case $C: u$ is a (standard or excess) station to the left of $v_{k}$ and $v$ is a junction or a station to the left of $v_{k}$. Let $S_{i}$ be the set of stations containing $u$; i.e., $u$ lies in the upper or lower grid between $v_{i}$ and $v_{i+1}$. The arguments in Cases A and B imply that $v_{i} \in W(v)$. If $v$ is also a station in $S_{i}$ or $v=v_{i+1}$ then it is easy to verify, by construction, that $W(u)$ and $W(v)$ are non-convergent (see Fig. 9); this also holds if $u$ and/or $v$ are excess stations. Suppose then that $v$ lies to the right of $v_{i+1}$. Consider first the case where $u$ is a standard station. Then both $v_{i}$ and $v_{i+1}$ lie in the left wedge of $W(v)$, and $u$ is incident to a line $\lambda$ that passes through $v_{i}$ and to a line $\lambda^{\prime}$ that passes through $v_{i+1}$. If $u \notin W(v)$ then a boundary line of $W(v)$ must separate $u$ from $v_{i}$ and $v_{i+1}$, in which case $v \in W(u)$; see Fig. 14(a).

Suppose next that $u$ is an excess station on the upper grid between $v_{i}$ and $v_{i+1}$. If $u \notin W(v)$ then $u$ must lie above $W(v)$. In this case $u$ is incident to a line $\lambda$ (an excess line) that passes through or below $v_{i}$ and to a line $\lambda^{\prime}$ that passes through $v_{i+1}$. As above, it is easily seen that the line through $v$ that bounds the left wedge of $W(v)$ from above must cross $\lambda$ to the left of $u$ and $\lambda^{\prime}$ to the right of $u$ and to the left of $v$, again implying that $v \in W(u)$; see Fig. 14(b).

A fully symmetric argument applies when $u$ is an excess station on the corresponding lower grid.

Note that Cases B and C also apply to excess stations constructed in the backtracking processes, starting either from some junction that precedes $v_{k}$ or from $v_{k}$ itself. 


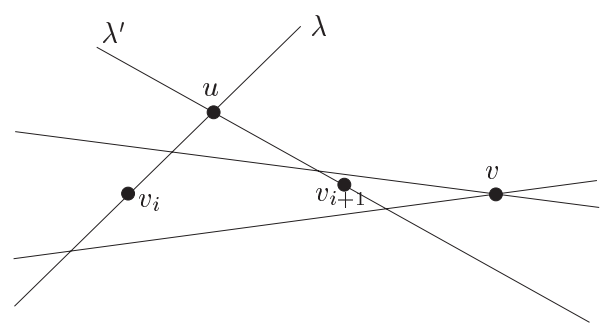

(a)

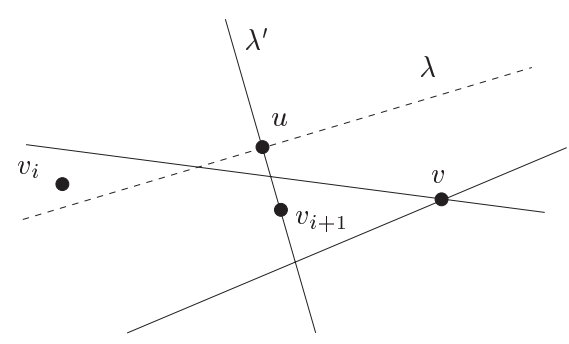

(b)

Fig. 14. Illustrating Case $C$ of the proof that $W(u)$ and $W(v)$ cannot be convergent. (a) $u$ is a standard station. (b) $u$ is an excess station on the upper grid.

Case D: $u$ is a junction and $v$ is a terminus to the right of $v_{k}$. Refer to Fig. 13 to recall the types of termini that we construct. Consider first the case where $v$ is the intersection point of an excess line $\lambda$ that passes through or below $v_{k}$, with either the line $\ell$ (in the case depicted in Fig. 13(a)), or the line $\ell^{\prime}$ (in the case depicted in Fig. 13(b)). By construction, $\lambda$ passes through or below $u$ and $\ell$ or $\ell^{\prime}$ passes through or above $u$, so $u \in W(v)$.

Consider next the case where $v$ is the intersection of $\ell$ with some line $\mu$ in $\Lambda_{k}^{+}$. Here too it is easily verified that $u$ lies between the two lines, so $u \in W(v)$. The same argument applies to the last possible case, where $v$ is the intersection of $\ell_{0}$ with some line of $\Lambda_{k}^{-}$.

Case E: $u$ is a station and $v$ is a terminus to the right of $v_{k}$. Let $S_{i}$ be the set of stations containing $u$. The arguments in Case D imply that $v_{i}, v_{i+1} \in W(v)$. If $u \notin W(v)$ then, arguing as in Case $\mathrm{C}$, we must have $v \in W(u)$.

Case F: Both $u$ and $v$ are termini to the right of $v_{k}$. This case follows from a direct inspection of all the possible types of pairs of termini; see Fig. 13.

This completes the proof of the claim, and thus of Theorem 3.1.

\section{Constructing the Sets of Segments $F$ and $E$ in the Plane $\pi$ and in 3-Space}

Consider the projected set $R$ of non-collinear points in the plane $\pi$, as defined in Section 2 , and recall that we assume that its total weight $w(R)=n-1$ is even. Recall also that we have partitioned $R$ into two sets, $R^{+}$and $R^{-}$, by some vertical line which we choose to be the $y$-axis. Instead of selecting the suitable set of segments $F$ in $\pi$, it is more convenient to work in the dual plane, using the same duality transform as in the proof of Theorem 3.1, where segments correspond to double wedges. First, we define the apices $v$ of these double wedges $W(v)$, that are vertices in the arrangement of lines dual to the elements of $R$, and then we specify the boundary lines of each $W(v)$, which are the duals of the endpoints of the corresponding segment $f(v)$ in the "primal" plane $\pi$.

The main part of the selection algorithm is an iterative pruning process that collects two types of different crossing points $v$, so-called junctions and stations, between the lines dual to the points of $R$. This process has many aspects similar to the one discussed in [14], and to the one given in the preceding section, but here the analysis is considerably 
more involved, because we have to handle weighted lines, and because the potential presence of the central bichromatic point $q$ further complicates certain steps of the analysis.

After associating each collected vertex $v$ with a certain double wedge $W(v)$ that has $v$ as an apex, we consider the set $F$ of segments $f(v)$ in the primal plane $\pi$ that correspond to these double wedges, by duality. Each segment $f(v)$ connects two elements of $R$ in $\pi$, and we show that these segments are pairwise non-convergent. Each segment $f(v) \in F$ spans with $p_{0}$ a plane $h(v)$ in $\mathbb{R}^{3}$, and we apply Theorem 3.1 to collect segments that connect pairs of points of $P$ within $h(v)$. We denote by $E(f(v))=E(v)$ (and sometimes also by $E(f)$ ) the set of segments in $\mathbb{R}^{3}$ that are spanned by $P$ and are determined (in this manner) by $f(v)$, and we set $E:=\bigcup_{f \in F} E(f)$.

\subsection{Collecting Junctions in the Dual Plane}

Denote by $L$ the set of lines dual to the elements of $R$. By choosing the directions of the coordinate frame sufficiently generic, we may assume that no two lines in $L$ are parallel. (In the primal plane $\pi$, this would correspond to the requirement that no two points of $R$ have the same $x$-coordinate.) Each line $\ell \in L$ has a weight $w(\ell)$ equal to the weight of its dual point, so $\sum_{\ell \in L} w(\ell)=n-1$. Let $L^{+}$and $L^{-}$denote respectively the sets of lines dual to $R^{+}$and $R^{-}$. Since we have assumed in Section 2 that $R^{+}$lies to the left of the $y$-axis and $R^{-}$lies to its right, it follows that all lines in $L^{-}$have negative slopes and all lines in $L^{+}$have positive slopes. The central bichromatic point $q$, when it exists, is mapped to a horizontal line $q^{*}$, which we assume to be the $x$-axis. This line appears as two coincident copies, $\left(q^{+}\right)^{*} \in L^{+}$and $\left(q^{-}\right)^{*} \in L^{-}$, with corresponding weights $w\left(q^{+}\right), w\left(q^{-}\right)$.

We begin by constructing a sequence $J=\left\langle v_{1}, v_{2}, \ldots, v_{k}\right\rangle$ of vertices of $\mathcal{A}(L)$, called junctions.

STEP 1. Set $i:=1$ and $L_{i}^{+}:=L^{+}, L_{i}^{-}:=L^{-}$.

STEP 2. If $L_{i}^{+}=L_{i}^{-}=\emptyset$, the construction of $J$ terminates. Otherwise, as we will see, neither set is empty. Let $v_{i}$ be the leftmost intersection point between a line in $L_{i}^{+}$and a line in $L_{i}^{-}$. Let $d_{i}^{+}, d_{i}^{-}$denote the overall weight of those lines of $L_{i}^{+}, L_{i}^{-}$, respectively, that are incident to $v_{i}$, and put $d_{i}:=\min \left\{d_{i}^{+}, d_{i}^{-}\right\}$. Suppose, without loss of generality, that $d_{i}=d_{i}^{+}$. Remove from $L_{i}^{+}$all its lines incident to $v_{i}$, and prune $L_{i}^{-}$as follows. Remove as many of the steepest lines of $L_{i}^{-}$(those with the smallest slopes) incident to $v_{i}$ as possible, so that their overall weight $c_{i}$ does not exceed $d_{i}$. If this weight is equal to $d_{i}$, we are done. Otherwise, we take the next steepest line $\ell$ and reduce its weight by $d_{i}-c_{i}$. The line $\ell$ is not removed from $L_{i}^{-}$. Note that each of the remaining lines of $L_{i}^{-}$incident to $v_{i}$ separates the removed lines of $L_{i}^{+}$from the removed lines of $L_{i}^{-}$. See Fig. 15. Set $L_{i+1}^{+}$and $L_{i+1}^{-}$to be the sets of surviving weighted lines of $L_{i}^{+}$and $L_{i}^{-}$, respectively, where the line $\ell$, if exists, has its new reduced weight. Set $i:=i+1$ and repeat Step 2.

Since $m_{1}$ is the line with the largest slope connecting a point of $R^{+}$and a point of $R^{-}$, our duality implies that $m_{1}^{*}$, the dual of $m_{1}$, is the leftmost intersection point between a line of $L^{+}$and a line of $L^{-}$. Hence, we have $v_{1}=m_{1}^{*}$. 


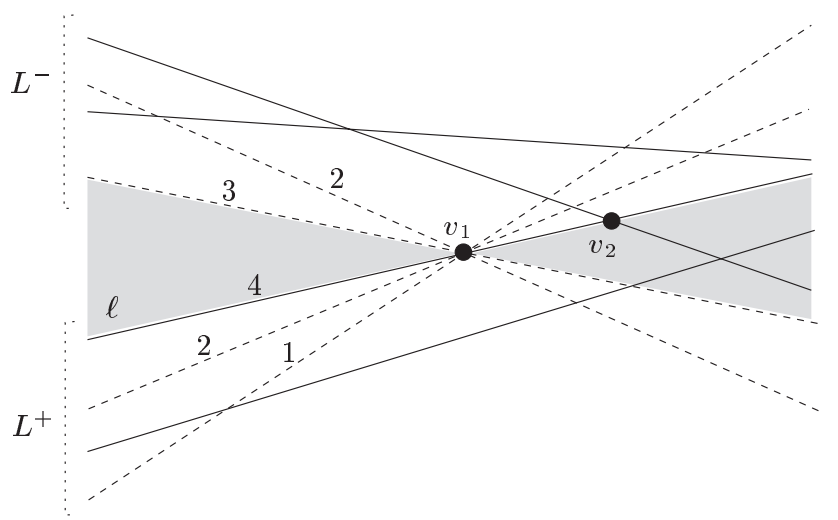

Fig. 15. Choosing the first junction $v_{1}$. Lines are labeled with their weight. We have $d_{1}=5$. The dashed lines, two from $L^{+}$and two from $L^{-}$, are removed, and the remaining line $\ell$ has its weight reduced by 2 at $v_{1}$. The double wedge $W\left(v_{1}\right)$ is shaded. The next junction $v_{2}$ is also shown.

If $q$ exists, then $v_{1}=m_{1}^{*}$ is the leftmost vertex along the line $q^{*}$ (see Fig. 3(ii)). At least one of the coincident copies $\left(q^{+}\right)^{*},\left(q^{-}\right)^{*}$ of $q^{*}$ contributes its full weight to $d_{1}$. Consequently, at least one of these copies is removed at $v_{1}$, which implies that $q^{*}$ belongs from this point on to only one of the sets $L_{i}^{+}, L_{i}^{-}$. In other words, the presence of $q$ will only affect the construction "in the vicinity" of $v_{1}$; see below for details.

As our construction sweeps the dual plane from left to right, we collect junctions (and stations) whose dual lines rotate clockwise from $m_{1}$ onwards (see Fig. 3).

As in the proof of Theorem 3.1, it is easy to verify the following properties of the above construction (consult Fig. 15):

\section{Claim 4.1.}

(i) $w\left(L_{i}^{+}\right)=w\left(L_{i}^{-}\right)$, for each $i=1, \ldots, k$.

(ii) For every $1 \leq i<j \leq k$, the junction $v_{i}$ lies in the left unbounded face $f_{j}$ of $\mathcal{A}\left(L_{j}^{+} \cup L_{j}^{-}\right)$that separates $L_{j}^{+}$and $L_{j}^{-}$at $x=-\infty$, and whose rightmost vertex is $v_{j}$. The point $v_{i}$ lies in the interior of $f_{j}$ if $d_{i}^{+}=d_{i}^{-}$; otherwise it may lie on the boundary of $f_{j}$.

(iii) $\sum_{i=1}^{k} d_{i}=(n-1) / 2$.

(iv) At the time when $v_{i}$ is constructed, the weights of all lines that are removed or weight-reduced at $v_{i}$, are equal to their original weights (i.e., before being reduced at any preceding junction), with the only possible exception of the two shallowest lines in their respective sets, whose weights could have been reduced earlier.

Proof of Claim 4.1(iv). Let $\ell^{+}$be a line of $L_{i}^{+}$that is removed at $v_{i}$ and is different from the shallowest such line $\ell_{a}^{+}$. Then, by property (ii), $\ell^{+}$must pass strictly below each of the previously constructed junctions, so it did not participate in any preceding pruning step. The argument for $L_{i}^{-}$is fully symmetric. 
We define, for each $1 \leq i \leq k$, the set of lines of $L_{i}^{+}$(resp., $L_{i}^{-}$) that are incident to $v_{i}$ and are either removed at $v_{i}$ or have their weight reduced there, by $D_{i}^{+}$(resp., $D_{i}^{-}$). We also put $D_{i}:=D_{i}^{+} \cup D_{i}^{-}$.

We associate with each junction $v_{i}$ the double wedge $W\left(v_{i}\right)$ bounded by the shallowest lines in $D_{i}^{+}$and $D_{i}^{-}$, respectively. See Fig. 15.

\subsection{Constructing Segments in E from the Junctions}

In the primal plane $\pi$, each junction $v_{j}$, for $j=1, \ldots, k$, corresponds to some line $v_{j}^{*}$ in $\pi$, which contains projections (from $p_{0}$ ) of some points of $P$. Let $h$ denote the plane spanned by $v_{j}^{*}$ and $p_{0}$. We apply Theorem 3.1 to a certain subset of $P \cap h$, thereby obtaining a set of pairwise non-convergent segments determined by the points in that subset.

The presence of the central bichromatic point $q$ may force us to modify the analysis at $v_{1}$. We first describe the analysis under the assumption that $q$ does not exist (we sometimes refer to this situation as the standard case), and then discuss the modifications needed at $v_{1}$ when $q$ exists.

The Case where $q$ Does not Exist. Fix an index $1 \leq i \leq k$. Let $\ell_{1}^{+}, \ldots, \ell_{a}^{+}$denote the lines of $D_{i}^{+}$, and let $\ell_{1}^{-}, \ldots, \ell_{b}^{-}$denote the lines of $D_{i}^{-}$. We enumerate the lines in the order of their slopes, so that $\ell_{a}^{+}$and $\ell_{b}^{-}$are the shallowest lines of $D_{i}^{+}$and $D_{i}^{-}$, respectively. Consider the line $v_{i}^{*}$ dual to $v_{i}$, and let $h$ be the plane spanned by $v_{i}^{*}$ and $p_{0}$. Let $X^{+}$(resp., $X^{-}$) denote the set of points of $P \backslash\left\{p_{0}\right\}$ whose projection from $p_{0}$ is one of the duals of $\ell_{1}^{+}, \ldots, \ell_{a}^{+}$(resp., $\ell_{1}^{-}, \ldots, \ell_{b}^{-}$) on $\pi$. By construction, $\left|X^{+}\right|,\left|X^{-}\right| \geq d_{i}$; either of $\left|X^{+}\right|$and $\left|X^{-}\right|$may consist of more than $d_{i}$ points, in the case when either $L_{i}^{+}$or $L_{i}^{-}$has a line whose weight is reduced (at $v_{i}$ or in some earlier junction). By Claim 4.1(iv), only $\ell_{a}^{+}$and $\ell_{b}^{-}$(which are the shallowest lines of $D_{i}^{+}$and $D_{i}^{-}$) may have reduced weight. By construction, the sets $X^{+}$and $X^{-}$are separated by a line in $h$ (consult with Fig. 7(a)).

Clearly, $\left|X^{+}\right|=\sum_{j=1}^{a} w\left(\ell_{j}^{+}\right)$and $\left|X^{-}\right|=\sum_{j=1}^{b} w\left(\ell_{j}^{-}\right)$, where the $w(\ell)$ 's denote the original weights of the corresponding lines $\ell$. We claim that Theorem 3.1 can be applied to the set $X=X^{+} \cup X^{-}$within the plane $h$. Indeed, assume without loss of generality that $\left|X^{+}\right| \geq\left|X^{-}\right|$. It follows from the construction that the points projecting to $\left(\ell_{a}^{+}\right)^{*}$ lie on the innermost ray from $p_{0}$ to $X$. Since $\ell_{a}^{+}$is either deleted at $v_{i}$ or has its weight reduced there, it follows that $\sum_{j=1}^{a-1} w\left(\ell_{j}^{+}\right)<\sum_{j=1}^{b} w\left(\ell_{j}^{-}\right)$. Therefore

$$
\left|X^{+}\right|-\left|X^{-}\right|=\sum_{j=1}^{a} w\left(\ell_{j}^{+}\right)-\sum_{j=1}^{b} w\left(\ell_{j}^{-}\right)<w\left(\ell_{a}^{+}\right) .
$$

Theorem 3.1 can thus be applied to the set $X=X^{+} \cup X^{-}$within the plane $h$, and it yields a total of at least $\left|X^{+}\right|+\left|X^{-}\right|+1$ pairwise non-convergent segments, each of which connects a point of $X^{+} \cup\left\{p_{0}\right\}$ to a point of $X^{-} \cup\left\{p_{0}\right\}$. However, there may exist one segment that has to be excluded because of potential collinearity with segments generated at other junctions: This is a segment $e$ along the ray from $p_{0}$ to the dual of the unique line $\ell$ among $\ell_{a}^{+}, \ell_{b}^{-}$whose weight is reduced at $v_{i}$ but which is not removed there, 
if such a line exists. (Note that this ray is the innermost among those rays connecting $p_{0}$ to points of the corresponding set $X^{+}$or $X^{-}$.) In that case, $\ell$ will also contribute weight to another subsequent junction $v_{i^{\prime}}$, where a segment collinear with $e$ may be generated in the primal plane, and these two segments cannot both be included in the output set $E$ (whose elements have to be pairwise non-convergent). Reducing the count due to this potential double counting, we are therefore left with at least

$$
w\left(D_{i}^{+}\right)+w\left(D_{i}^{-}\right)+1-\zeta_{i}=\sum_{j=1}^{a} w\left(\ell_{j}^{+}\right)+\sum_{j=1}^{b} w\left(\ell_{j}^{-}\right)+1-\zeta_{i}
$$

pairwise non-convergent segments, where $\zeta_{i}=1$ if there is a line whose weight has been reduced at $v_{i}$ but which was not removed there, and $\zeta_{i}=0$ otherwise. Here $w\left(D_{i}^{+}\right)$, $w\left(D_{i}^{-}\right)$denote the total original weight of these sets.

Handling the Central Bichromatic Point $q$. As noted, the presence of $q$ may force us to modify the analysis at the first junction $v_{1}$, because the dual line $q^{*}$ appears there as two coincident lines $\left(q^{-}\right)^{*} \in L_{1}^{-}$and $\left(q^{+}\right)^{*} \in L_{1}^{+}$. Let $d_{0}^{+}$(resp., $d_{0}^{-}$) denote the total weight of all the lines of $D_{1}^{+} \backslash\left\{\left(q^{+}\right)^{*}\right\}$ (resp., of $D_{1}^{-} \backslash\left\{\left(q^{-}\right)^{*}\right\}$ ); recall that at least one of the sets $D_{1}^{+}, D_{1}^{-}$includes the respective copy of $q^{*}$ with its full weight. We have $d_{1}=\min \left\{d_{0}^{+}+w\left(q^{+}\right), d_{0}^{-}+w\left(q^{-}\right)\right\}$; assume, without loss of generality, that $d_{1}=d_{0}^{+}+w\left(q^{+}\right) \leq d_{0}^{-}+w\left(q^{-}\right)$.

Suppose first that $d_{0}^{-} \geq d_{0}^{+}+w\left(q^{+}\right)$. Refer to Fig. 16. Then $\left(q^{-}\right)^{*} \notin D_{1}^{-}$. Let $X^{-}$ denote the set of all points $p \in P \backslash\left\{p_{0}\right\}$ that project to the points dual to the lines of $D_{1}^{-}$. If $D_{1}^{-}$contains a line $\ell$ whose weight is only reduced at $v_{1}$, let $b_{1}>0$ denote the surviving weight of $\ell$; otherwise, put $b_{1}=0$. We have $\left|X^{-}\right|=d_{1}+b_{1}$. Let $X^{+}$denote the set of all points $p \in P \backslash\left\{p_{0}\right\}$ that project to the points dual to the lines of $D_{1}^{+}$, including $q$ (with its full weight $\left.w(q)=w\left(q^{-}\right)+w\left(q^{+}\right)\right)$. We have $\left|X^{+}\right|=d_{1}+w\left(q^{-}\right)$. If $b_{1} \leq w\left(q^{-}\right)$, then $\left|X^{+}\right|-\left|X^{-}\right|=w\left(q^{-}\right)-b_{1}<w(q)$; the right-hand side is the number of points on the innermost ray from $p_{0}$ to the points of $X^{+}$(see Fig. 16). If $b_{1}>w\left(q^{-}\right)$, then $\left|X^{-}\right|-\left|X^{+}\right|=b_{1}-w\left(q^{-}\right)<w(\ell)$; the right-hand side is the number of points on the innermost ray from $p_{0}$ to the points of $X^{-}$. Hence, in either case, Theorem 3.1 is

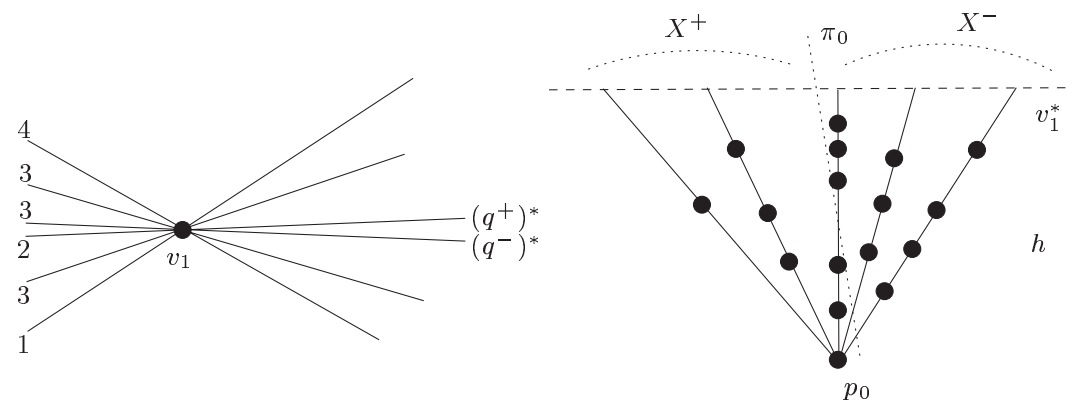

Fig. 16. Collecting segments in $E\left(v_{1}\right)$ when $q$ exists. Here $\left(q^{-}\right)^{*} \notin D_{1}^{-}$. We have $d_{1}=6,\left|X^{-}\right|=7$, and $\left|X^{+}\right|=9$. The lines $\left(q^{-}\right)^{*}$ and $\left(q^{+}\right)^{*}$ are coincident, but are drawn as separate lines for the purpose of illustration. 

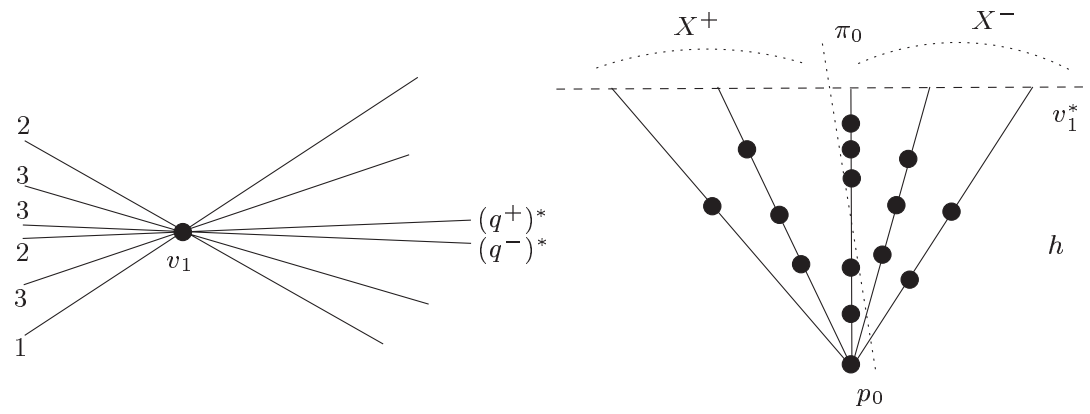

Fig. 17. Collecting segments in $E\left(v_{1}\right)$ when $q$ exists. Here $\left(q^{-}\right)^{*} \in D_{1}^{-}, d_{1}=6,\left|X^{-}\right|=5,\left|X^{+}\right|=9$, and Theorem 3.1 can be applied.

applicable to $X^{+} \cup X^{-} \cup\left\{p_{0}\right\}$, and it yields a set $E\left(v_{1}\right)$ of at least

$$
w\left(D_{1}^{-}\right)+w\left(D_{1}^{+} \backslash\{q\}\right)+w(q)+1=w\left(D_{1}^{-}\right)+w\left(D_{1}^{+}\right)+w\left(q^{-}\right)+1
$$

pairwise non-convergent segments, where, as above, each line in $D_{1}^{-} \cup D_{1}^{+}$is taken with its full original weight. Compared with the count in the standard case, we collect $w\left(q^{-}\right)$ additional segments in this case.

Suppose next that $d_{0}^{-}<d_{0}^{+}+w\left(q^{+}\right) \leq d_{0}^{-}+w\left(q^{-}\right)$. See Fig. 17. In this case, $D_{1}^{-}$ contains $\left(q^{-}\right)^{*}$ and $D_{1}^{+}$contains $\left(q^{+}\right)^{*}$. We let $X_{0}^{+}$(resp., $X_{0}^{-}$) denote the set of all points of $P \backslash\left\{p_{0}\right\}$ that project to the points dual to the lines of $D_{1}^{+} \backslash\left\{\left(q^{+}\right)^{*}\right\}$ (resp., the lines of $\left.D_{1}^{-} \backslash\left\{\left(q^{-}\right)^{*}\right\}\right)$. We have $\left|X_{0}^{+}\right|=d_{0}^{+}$and $\left|X_{0}^{-}\right|=d_{0}^{-}$.

First, assume further that $d_{0}^{+} \neq d_{0}^{-}$, say $d_{0}^{+}>d_{0}^{-}$. In this case we set $X^{+}:=X_{0}^{+}$ and take $X^{-}$to be the union of $X_{0}^{-}$with the set of all points of $P \backslash\left\{p_{0}\right\}$ that project to $q$. We have $\left|X^{-}\right|=d_{0}^{-}+w(q)>\left|X^{+}\right|$and $\left|X^{-}\right|-\left|X^{+}\right|=d_{0}^{-}-d_{0}^{+}+w(q)<w(q)$. A symmetric argument holds when $d_{0}^{+}<d_{0}^{-}$. Hence, Theorem 3.1 is again applicable, and yields a set $E\left(v_{1}\right)$ of at least

$$
d_{0}^{-}+d_{0}^{+}+w(q)+1 \geq w\left(D_{1}^{+}\right)+w\left(D_{1}^{-}\right)+1
$$

pairwise non-convergent segments, where, as above, each line in $D_{1}^{-} \cup D_{1}^{+}$is taken with its full original weight. Here the lower bound is the same as the one yielded in the standard case.

The final, problematic case arises when $d_{0}^{+}=d_{0}^{-}$. See Fig. 18. In this case the points of $P \backslash\left\{p_{0}\right\}$ that project to $q$ can be added to either set $X_{0}^{-}$or $X_{0}^{+}$, say we add them to $X_{0}^{-}$. Then the resulting sets $X^{-}, X^{+}$satisfy $\left|X^{+}\right|=d_{0}^{+},\left|X^{-}\right|=d_{0}^{-}+w(q)$, and $\left|X^{-}\right|-\left|X^{+}\right|=w(q)$. In this case Theorem 3.1 is not applicable, and we can only apply Corollary 3.2, to obtain a set $E\left(v_{1}\right)$ with at least

$$
d_{0}^{-}+d_{0}^{+}+w(q) \geq w\left(D_{1}^{+}\right)+w\left(D_{1}^{-}\right)
$$

pairwise non-convergent segments. That is, we lose one segment in $E\left(v_{1}\right)$, compared with the standard situation. (Note that in this case all lines through $v_{1}$ are removed, except perhaps for $\left(q^{-}\right)^{*}$.) 


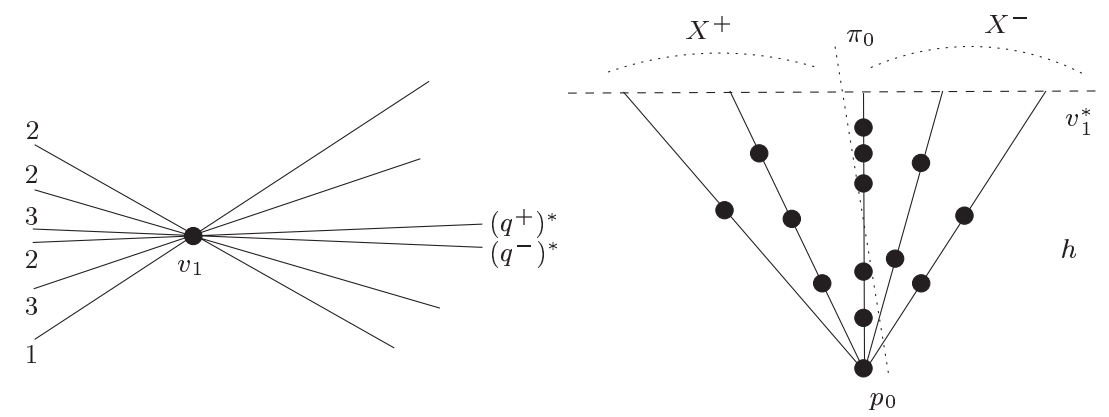

Fig. 18. Collecting segments in $E\left(v_{1}\right)$ when $q$ exists. Here $\left(q^{-}\right)^{*} \in D_{1}^{-}, d_{1}=6,\left|X^{-}\right|=4,\left|X^{+}\right|=9$, and $\left|X^{+}\right|-\left|X^{-}\right|=w(q)$, so only Corollary 3.2 can be applied.

In addition, as in the standard case, we need to subtract 1 from any of the bounds obtained above, in case $D_{1}^{+}$or $D_{1}^{-}$has a line whose weight is only reduced at $v_{1}$, to accommodate the potential double counting due to collinear segments generated at subsequent junctions.

The double wedge $W\left(v_{1}\right)$ associated with $v_{1}$ is defined as in the standard case, except that in some of the above cases it may degenerate to the single line $q^{*}$ (it always has $\left(q^{-}\right)^{*}$ or $\left(q^{+}\right)^{*}$ as one of its bounding lines). In this case we still consider $W\left(v_{1}\right)$ to have its apex at $v_{1}$. In the primal plane the corresponding segment $f\left(v_{1}\right)$ degenerates to the singleton point $q$, but it is still considered to lie along the line $v_{i}^{*}$.

Wrapping Up. We repeat this collection process to each of the junctions $v_{i}$, and sum up the resulting bounds. This sum can be rearranged as follows. Let $\ell_{1}, \ldots, \ell_{t}$ denote an enumeration of all the lines in $L$, and put $w_{j}=w\left(\ell_{j}\right)$ (the original weight), for $j=1, \ldots, t$. (In case $q$ exists, the lines $\left(q^{+}\right)^{*}$ and $\left(q^{-}\right)^{*}$ appear as two separate lines in this enumeration, with their respective weights.) For each $j$, let $\kappa_{j}$ denote the number of junctions $v_{s}$ that are incident to $\ell_{j}$, such that $\ell_{j} \in D_{s}$. Observe that if $\kappa_{j}>1$, then in the first $\kappa_{j}-1$ of these junctions $v_{s}$, the weight of $\ell_{j}$ is reduced at $v_{s}$ but $\ell_{j}$ is not removed there. Hence $\zeta_{s}=1$ at each of these junctions $v_{s}$, and we "blame" this reduction in the count on $\ell_{j}$, making its effective weight contribution at $v_{s}$ equal to $w_{j}-1 . \ell_{j}$ is removed only at the last (i.e., the $\kappa_{j}$ th) of these junctions. Therefore, the overall number of segments in $E$ generated at all the junctions $v_{1}, \ldots, v_{k}$ is at least

$$
k+\sum_{j=1}^{t}\left(\kappa_{j}-1\right)\left(w_{j}-1\right)+\sum_{j=1}^{t} w_{j}-\varepsilon_{0}=k+t+\sum_{j=1}^{t} \kappa_{j}\left(w_{j}-1\right)-\varepsilon_{0},
$$

where $\varepsilon_{0}=1$ if $q$ exists and the problematic case $d_{0}^{-}=d_{0}^{+}$arises at $v_{1}$, and $\varepsilon_{0}=0$ in all other cases.

\subsection{Collecting Stations in the Dual Plane and Corresponding Segments in E}

In the next step we collect additional vertices, called stations, between pairs of successive junctions $v_{j}, v_{j+1}$. We first handle the standard case, in which either $q$ does not exist, or 


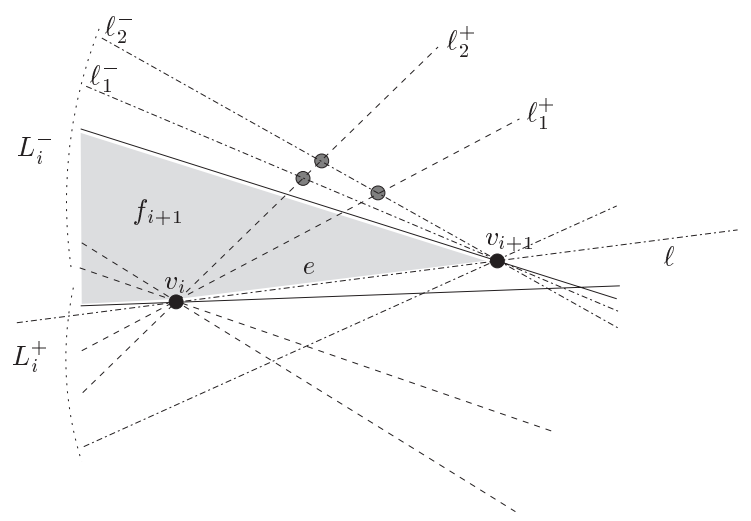

Fig. 19. Collecting stations (shown encircled) between $v_{i}$ and $v_{i+1}$. The dashed lines are those removed at $v_{i}$, and the dashed-dotted ones are those removed at $v_{i+1}$.

$q$ exists and $j \geq 2$, and then present a modified analysis for the case where $q$ exists and $j=1$.

The Standard Case. Fix an index $1 \leq i<k$, and consider the vertical slab between $v_{i}$ and $v_{i+1}$. By Claim 4.1(ii), $v_{i}$ lies inside or on the boundary of the face $f=f_{i+1}$ of $\mathcal{A}\left(L_{i+1}^{+} \cup L_{i+1}^{-}\right)$whose rightmost vertex is $v_{i+1}$; see Fig. 19. Hence, the segment $e=v_{i} v_{i+1}$ is contained in (the closure of) $f$. We distinguish two cases:

Case 1: $e$ is contained in the interior of $f$ (except for its right endpoint). This implies that the lines of $D_{i+1}^{-}$(resp., of $D_{i+1}^{+}$) pass strictly above (resp., below) $e$. Moreover, either all the lines of $D_{i}^{-}$pass below $e$, or all the lines of $D_{i}^{+}$pass above $e$. Suppose, without loss of generality, that the second case arises. Denote the lines of $D_{i}^{+}$by $\ell_{1}^{+}, \ldots, \ell_{v_{i}}^{+}$, ordered according to increasing slope, and those of $D_{i+1}^{-}$by $\ell_{1}^{-}, \ldots, \ell_{v_{i+1}}^{-}$, ordered according to decreasing slope. See Fig. 19 (which depicts this configuration, even though it illustrates the following Case 2).

Each of the lines $\ell_{s}^{+}$intersects every line $\ell_{t}^{-}$in the slab between $v_{i}$ and $v_{i+1}$, because $\ell_{s}^{+}$passes through the left endpoint of $e, \ell_{t}^{-}$passes through the right endpoint of $e$, and they both lie above $e$. We refer to the points of intersection between these two sets of lines as the upper grid between $v_{i}$ and $v_{i+1}$; the lower grid is defined analogously. Consider the vertices of $\mathcal{A}(L)$ where $\ell_{v_{i}}^{+}$intersects the lines $\ell_{1}^{-}, \ldots, \ell_{v_{i+1}}^{-}$, and the vertices where $\ell_{v_{i+1}}^{-}$intersects the lines $\ell_{1}^{+}, \ldots, \ell_{v_{i}}^{+}$. There are $v_{i}+v_{i+1}-1$ distinct vertices of this kind (see Fig. 19), and we let the set of stations $S_{i}$ consist of all these vertices. We associate with each station $u$ the double wedge $W(u)$ between the two lines from $D_{i}^{+}$and $D_{i+1}^{-}$ that meet at $u$.

Each station $u$ generates a set $E(u)$ of segments spanned by $P$ in $\mathbb{R}^{3}$, as follows. Suppose that $u$ is incident to some line $\ell_{s}^{+}$through $v_{i}$ and to some line $\ell_{t}^{-}$through $v_{i+1}$ (where either $s=v_{i}$ or $t=v_{i+1}$ ). Consider the primal line $u^{*}$ dual to $u$, and let $h$ denote the plane in 3 -space spanned by $p_{0}$ and $u^{*}$. The plane $h$ contains two segments that connect $p_{0}$ to the two respective dual points $\left(\ell_{s}^{+}\right)^{*},\left(\ell_{t}^{-}\right)^{*}$, both lying on $u^{*}$. The first segment $\sigma^{+}$contains $w\left(\ell_{s}^{+}\right)$points of $R^{+}$, and the second segment $\sigma^{-}$contains 


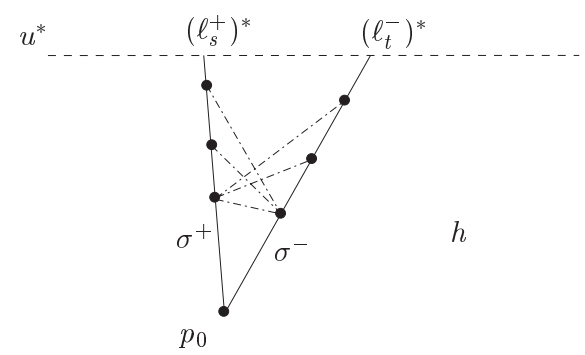

Fig. 20. The set $E(u)$ of segments (drawn dashed-dotted) spanned by $P$ that are determined by a station $u$.

$w\left(\ell_{t}^{-}\right)$points of $R^{-}$. We can easily collect here as many as $w\left(\ell_{p}^{+}\right)+w\left(\ell_{q}^{-}\right)-1$ segments into $E(u)$, no two of which are convergent; for example, one can get that many distinct segments by taking all segments one of whose endpoints is either the nearest point to $p_{0}$ on $\sigma^{+}$or the nearest point to $p_{0}$ on $\sigma^{-}$; see Fig. 20 .

These segments constitute the set $E(u)$. Hence, the total number of segments that are collected in this manner for all the new stations $u$ is

$$
\left|\bigcup_{u \in S_{i}} E(u)\right|=\sum_{t=1}^{v_{i+1}}\left[w\left(\ell_{v_{i}}^{+}\right)+w\left(\ell_{t}^{-}\right)-1\right]+\sum_{s=1}^{v_{i}-1}\left[w\left(\ell_{s}^{+}\right)+w\left(\ell_{v_{i+1}}^{-}\right)-1\right] .
$$

Note that the sum $\sum_{s=1}^{v_{i}} w\left(\ell_{s}^{+}\right)$is at least $d_{i}$; it may exceed $d_{i}$ if it involves a nondeleted line with reduced weight, because in the sum we use the full weight of that line. Similarly, $\sum_{t=1}^{v_{i+1}} w\left(\ell_{t}^{-}\right) \geq d_{i+1}$. Therefore, the total number of segments that we collect this way is at least

$$
d_{i}+d_{i+1}-1
$$

We note that this estimate is rather conservative. In general, if the weights of the lines are greater than 1 and $v_{i}, v_{i+1}>1$, we get a larger lower bound.

Case 2: $e$ is an edge of $f$. In this case, $e$ is contained in a line $\ell$ which is incident to $v_{i}$ but which was not removed when $v_{i}$ was constructed (it could have been the one whose weight has been reduced there). Assume first that $\ell$ is not the line whose weight has been reduced at $v_{i}$. By construction, it then follows that the lines of $D_{i}^{-}$pass strictly below $e$, and the lines of $D_{i}^{+}$pass strictly above $e$. Now either all the lines of $D_{i+1}^{-}$pass above $e$, or all the lines of $D_{i+1}^{+}$pass below $e$. We can now repeat the preceding arguments, and obtain, as above, a set $S_{i}$ of stations of $\mathcal{A}(L)$ along either the upper or the lower grid, which generate a total of at least $d_{i}+d_{i+1}-1$ segments spanned by $P$, which are added to $E$. Figure 19 depicts this case of the analysis.

Suppose next that the line $\ell$ containing $e$ is the (unique) weight-reduced line at $v_{i}$. If $\ell$ does not belong to $D_{i+1}$, then the first case of the analysis applies, and yields the same lower bound of $d_{i}+d_{i+1}-1$ on the number of collected segments that are added to $E$. We thus assume that $\ell$ does belong to $D_{i+1}$.

Let $a_{i}$ and $a_{i+1}$ denote the contribution of $\ell$ to $d_{i}$ and $d_{i+1}$, respectively. That is, the overall weight of the lines from the same family of $\ell$ (i.e., $L^{+}$or $L^{-}$) that are removed at $v_{i}$ (resp., at $\left.v_{i+1}\right)$ is $c_{i}=d_{i}-a_{i}$ (resp., $c_{i+1}=d_{i+1}-a_{i+1}$ ). 


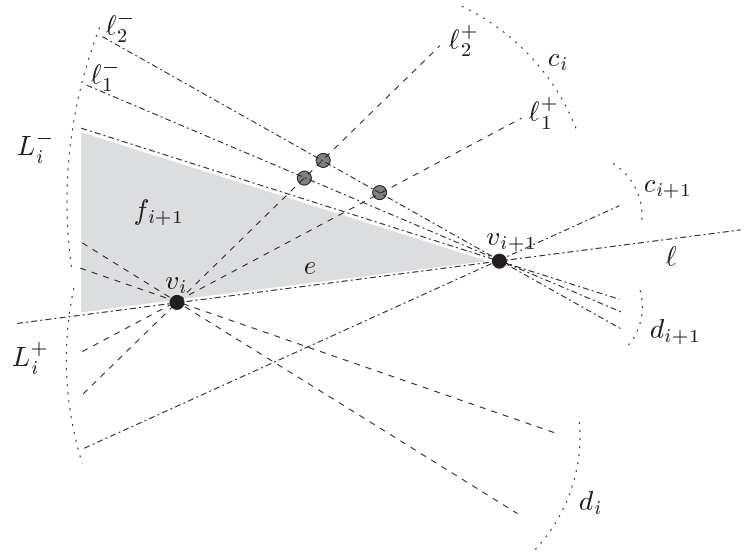

Fig. 21. Illustrating the proof of Claim 4.2.

Claim 4.2. In this case one can construct stations along either the upper or the lower grid between $v_{i}$ and $v_{i+1}$, from which at least

$$
c_{i}+c_{i+1}=d_{i}+d_{i+1}-\left(a_{i}+a_{i+1}\right)
$$

new segments can be collected in $E$ (in the same manner as before).

Proof. Indeed, suppose, without loss of generality, that $\ell \in L^{+}$. Then the total weight of the lines of $L^{-}$that are incident to $v_{i}$ (resp., to $v_{i+1}$ ) is $d_{i}$ (resp., $d_{i+1}$ ), and the total weight of the lines of $L^{+}$that are incident to $v_{i}$ (resp., to $v_{i+1}$ ) and are removed there is $c_{i}$ (resp., $c_{i+1}$ ). See Fig. 21.

If both $c_{i}$ and $c_{i+1}$ are 0 , the claim is trivial, so assume that, say, $c_{i}>0$ (see Fig. 21). In this case the upper grid between $v_{i}$ and $v_{i+1}$ exists, and generates, arguing as above, at least

$$
c_{i}+d_{i+1}-1=d_{i}+d_{i+1}-a_{i}-1 \geq d_{i}+d_{i+1}-\left(a_{i}+a_{i+1}\right)
$$

new segments in $E$, as claimed. The case where $c_{i+1}>0$ (and $c_{i}=0$ ) is fully symmetric, except that in this case we use the lower grid (see Fig. 21). This establishes our claim.

We denote by $E\left(S_{i}\right)$ the set of segments in $E$ that are constructed from the stations collected between the two consecutive junctions $v_{i}$ and $v_{i+1}$.

We have thus showed that $\left|E\left(S_{i}\right)\right| \geq d_{i}+d_{i+1}-1$, if there is no line that contributes weight to both junctions. If on the other hand there is a line $\ell$ that contributes a weight of $a_{i} \geq 1$ to $v_{i}$ and a weight of $a_{i+1} \geq 1$ to $v_{i+1}$, then $\left|E\left(S_{i}\right)\right| \geq d_{i}+d_{i+1}-a_{i}-a_{i+1}$.

The Case where $q$ Exists and $j=1$. Suppose next that the central bichromatic point $q$ exists, and consider the construction of $S_{1}$. If $q^{*}$ does not pass through $v_{2}$, then the analysis proceeds in essentially the same manner as in the standard case. For the sake of completeness, we repeat the details. Suppose, without loss of generality, that $q^{*}$ passes below $v_{2}$. The case where $v_{1} v_{2}$ is interior to the face $f_{2}$ is handled in exactly the same 


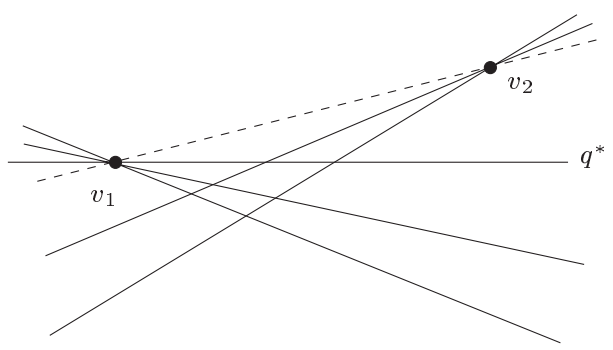

Fig. 22. Collecting stations in $S_{1}$ when $q^{*}$ passes below $v_{2}$.

way as above: One can use the lower grid between $v_{1}$ and $v_{2}$, and construct stations that contribute a total of at least $d_{1}+d_{2}-1$ segments to $E$; see Fig. 22. The same holds for the case where there is a line (different from $q^{*}$ ) that passes through both $v_{1}$ and $v_{2}$ but it belongs to at most one of the sets $D_{1}, D_{2}$ (in this case, one of the upper or lower grids will generate stations with $\left.\left|E\left(S_{1}\right)\right| \geq d_{1}+d_{2}-1\right)$. Consider then the case where there exists such a line $\ell$ whose weight has been reduced at both junctions (it may have been removed at $v_{2}$ ). Since the entire $L^{-}$-weight of $q$ must have been removed at $v_{1}$ (because no surviving line of $L^{-}$can pass below $v_{2}$ ), it follows that $\ell \in L_{1}^{+}$. Again, this case can be handled as in Claim 4.2, and yields at least $d_{1}+d_{2}-a_{1}-a_{2}$ in $E$, according to the preceding notation. In summary, we can always collect from the stations in $S_{1}$ at least either $d_{1}+d_{2}-1$ or $d_{1}+d_{2}-a_{1}-a_{2}$ segments into $E$, depending on the cases considered above. (Note that in some of these cases we may actually gain $w\left(q^{+}\right)$ additional segments in $E\left(S_{1}\right)$.)

Assume then that $q^{*}$ passes through $v_{2}$. Without loss of generality assume that $\left(q^{+}\right)^{*}$ is fully removed at $v_{1}$. The following cases can arise:

Case 1. $\left(q^{-}\right)^{*} \in D_{2}^{-}$. Let $a_{1}$ (resp., $a_{2}$ ) denote the weight removed from $\left(q^{-}\right)^{*}$ at $v_{1}$ (resp., at $v_{2}$ ). (It is possible that $a_{1}=0$.) We claim that one can collect at least $d_{1}+d_{2}-\left(a_{1}+a_{2}\right)$ segments into $E\left(S_{1}\right)$ in either the upper or lower grid between $v_{1}$ and $v_{2}$. This is argued in much the same way as in the case where $q$ does not exist. Specifically, let $c_{1}=d_{1}-a_{1}$ (resp., $c_{2}=d_{2}-a_{2}$ ) denote the $L^{-}$-weight removed at $v_{1}$ (resp., $v_{2}$ ) from the lines in $L^{-}$other than $\left(q^{-}\right)^{*}$.

If both $c_{1}$ and $c_{2}$ are 0 , then there is nothing to prove. If $c_{1}>0$, then, except for $\left(q^{-}\right)^{*}$, all other lines of $D_{1}^{-}$pass strictly below $v_{2}$, and have total weight at least $c_{1}=d_{1}-a_{1}$. By assumption, all lines of $D_{2}^{+}$pass strictly below $v_{1}$, and have total weight at least $d_{2}$. Therefore the lower grid between $v_{1}$ and $v_{2}$ can be used to produce at least $c_{1}+d_{2}-1=d_{1}+d_{2}-1-a_{1} \geq d_{1}+d_{2}-\left(a_{1}+a_{2}\right)$ segments in $E\left(S_{1}\right)$.

If $c_{1}=0$ and $c_{2}>0$, then, except for $\left(q^{-}\right)^{*}$, all other lines of $D_{2}^{-}$pass strictly above $v_{1}$, and have total weight at least $c_{2}=d_{2}-a_{2}$. As $c_{1}=0$, there are no lines from $L^{-}$through $v_{1}$ other than $\left(q^{-}\right)^{*}$, and therefore at least one additional line (other than $\left.\left(q^{+}\right)^{*}\right)$ of $L^{+}$must pass through $v_{1}$ (or else $v_{1}$ would not be a vertex of the arrangement). Therefore, the upper grid between $v_{1}$ and $v_{2}$ exists, and we may use it to collect at least $1+c_{2}-1=c_{2}=d_{2}-a_{2}=\left(d_{1}-a_{1}\right)+d_{2}-a_{2}=d_{1}+d_{2}-\left(a_{1}+a_{2}\right)$ segments into $E\left(S_{1}\right)$. 


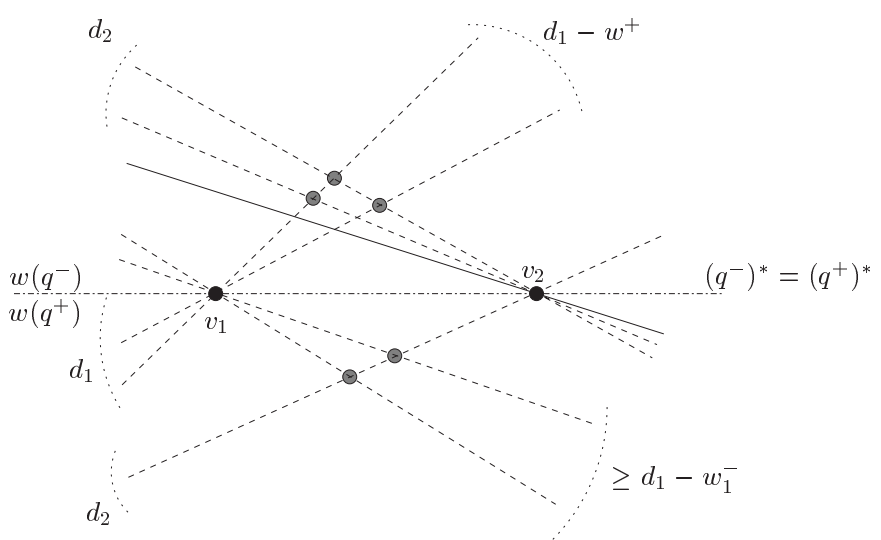

Fig. 23. The problematic case in the construction of $S_{1}$ in the presence of a central bichromatic point $q$ : Here $d_{1}=d_{1}^{+}$, so $\left(q^{+}\right)^{*}$ is removed at $v_{1}$. We also assume that $\left(q^{-}\right)^{*} \notin D_{2}^{-}$. In this case we can only guarantee the generation of at least $d_{1}+d_{2}-\max \left\{w^{+}, w_{1}^{-}\right\}-1$ segments in $E$, and we lose $\max \left\{w^{+}, w_{1}^{-}\right\}$segments in the bound.

Case 2. $\left(q^{-}\right)^{*} \notin D_{2}^{-}$(but it still passes through $v_{2}$ ). In this case, depicted in Fig. 23, the sets $D_{2}^{+}, D_{2}^{-}$are both non-empty, and all lines of $D_{2}^{+}$(resp., $D_{2}^{-}$) pass strictly below (resp., above) $v_{1}$; the total weight of either set is $d_{2}$. Set $w^{+}=w\left(q^{+}\right), w^{-}=w\left(q^{-}\right)$. There must exist either lines of $L^{+}$or lines of $L^{-}$(other than $q^{*}$ ) that pass through $v_{1}$ and are removed there. In the former case the total removed $L^{+}$-weight of these lines is $d_{1}-w^{+}$, and we may use the upper grid between $v_{1}$ and $v_{2}$ (which necessarily exists) to collect at least $d_{1}+d_{2}-w^{+}-1$ segments into $E\left(S_{1}\right)$. In the latter case, arguing in an essentially symmetric manner, we may use the lower grid between $v_{1}$ and $v_{2}$ (which necessarily exists) to collect at least $d_{1}+d_{2}-w_{1}^{-}-1$ segments into $E\left(S_{1}\right)$, where $w_{1}^{-}$ is the weight that $\left(q^{-}\right)^{*}$ contributes at $v_{1}$.

As will follow from the later counting phase, given in Section 4.4, we need to compensate for the loss of either $w^{+}$or $w_{1}^{-}$segments in $E\left(S_{1}\right)$, which we do by including the points on the ray $p_{0} q$ in the set $X^{+}$when we construct $E\left(v_{2}\right)$, even though neither $\left(q^{+}\right)^{*}$ nor $\left(q^{-}\right)^{*}$ belongs to $D_{2}$. Before doing so, the size of $X^{+}$is exactly $d_{2}$, and the size of $X^{-}$is at least $d_{2}$; it can be larger if there is a negative line whose weight is only reduced at $v_{2}$. We add the points on the ray $p_{0} q$ to $X^{+}$. In general, we can apply Theorem 3.1 to the modified sets $X^{-}, X^{+}$, except when $\left|X^{-}\right|=\left|X^{+}\right|=d_{2}$, in which case we can only apply Corollary 3.2. The modified $E\left(v_{2}\right)$ thus consists of at least $w\left(D_{2}^{+}\right)+w\left(D_{2}^{-}\right)+w(q)+1=2 d_{2}+w(q)+1$ pairwise non-convergent segments, if $\left|X^{-}\right| \neq\left|X^{+}\right|$, or of at least $w\left(D_{2}^{+}\right)+w\left(D_{2}^{-}\right)+w(q)=2 d_{2}+w(q)$ pairwise nonconvergent segments, if $\left|X^{-}\right|=\left|X^{+}\right|$. In the most pessimistic scenario, we can only apply Corollary 3.2, whereas, when $q$ was not included, we could have applied Theorem 3.1 to collect $2 d_{2}+1$ segments in $E\left(v_{2}\right)$. We thus gain at least $w(q)-1$ additional segments. However, we may have to subtract one extra segment from the count, because $\left(q^{-}\right)^{*}$ may contribute weight to a further junction. Thus, in the worst case, we can only guarantee $w(q)-2=w^{+}+w^{-}-2$ additional segments. In general, these suffice to compensate for the loss of $\max \left\{w^{+}, w_{1}^{-}\right\}$a $E\left(S_{1}\right)$, unless $\min \left\{w^{+}, w_{1}^{-}\right\}=1$. In this special case we lose one segment in our count. 


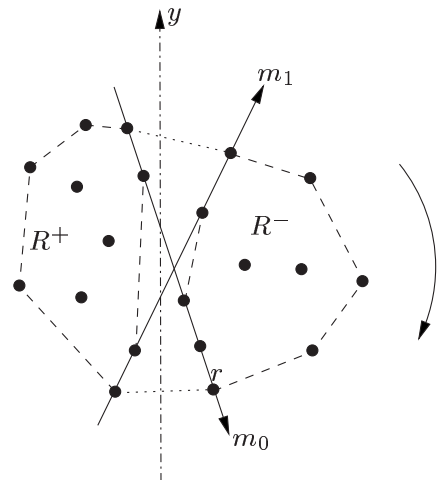

(i)

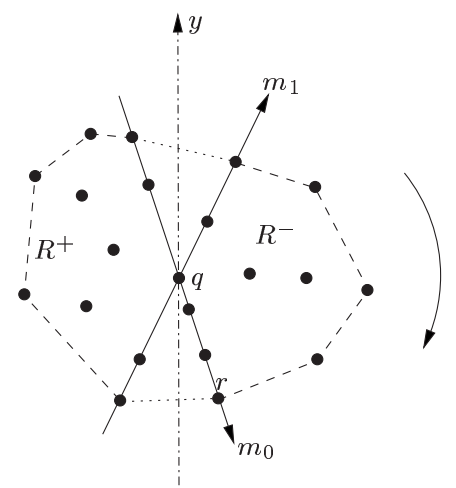

(ii)

Fig. 24. Reproducing the primal construction of $R^{-}$and $R^{+}$: Cases (i) and (ii).

The price that we pay for including $q$ is that the double wedge $W\left(v_{2}\right)$ has to shrink, and be bounded by $q^{*}$ and by the shallowest line in $D_{2}^{-}$. However, as we will later show, in Section 4.5, the collected double wedges will remain pairwise non-convergent.

Collecting Stations to the Left of $v_{1}$ and to the Right of $v_{k}$. We next define the last set of stations $S_{k}$, which are stations that lie to the right of $v_{k}$ or to the left of $v_{1}$. Recall the specific partition of $R$ into $R^{+}$and $R^{-}$, as presented in Section 2 . We will exploit certain features of this partition in the construction of $S_{k}$, and will find it convenient to "flip" between the primal and dual settings as we go. For the convenience of the reader, we reproduce here Fig. 3 as Fig. 24.

Claim 4.3. At least one of the following two conditions will be satisfied:

(i) The last junction, $v_{k}$, is identical to $m_{0}^{*}$, the dual of $m_{0}$.

(ii) $r^{*}$, the dual of $r \in R^{-}$, passes through $v_{k}$ and is the unique element of $L^{-}$deleted during the procedure at $v_{k}$.

Proof. Suppose that during the procedure $r^{*}$ is deleted at a junction $v_{j}$, for some $j \leq k$. Clearly, $v_{j}^{*}$ passes through $r$ and at least one point $t \in R^{+}$, whose dual line is also deleted, or has its weight reduced, at $v_{j}^{*}$.

If, in the primal plane $\pi, v_{j}^{*}$ passes through another point $r^{\prime} \neq r$ of $R^{-}$, then $v_{j}^{*}=m_{0}$ (otherwise it has to lie clockwise to $m_{0}$ and then it cannot meet any point of $R^{+}$). In this case, in the dual plane there cannot be any intersection point between a line of $L^{-}$and a line of $L^{+}$to the right of $v_{j}$, so that $j=k$. That is, we have $v_{k}^{*}=m_{0}$, and (i) holds.

Suppose then that, in the primal plane $\pi, v_{j}^{*}$ does not pass through any element $r^{\prime} \in R^{-}$ other than $r$. If $j=k$, then condition (ii) is satisfied. So we can assume that $j<k$ and $v_{k}^{*} \neq m_{0}$. Refer to Fig. 25. Take any two lines $\ell_{-} \in L^{-}$and $\ell_{+} \in L^{+}$in the dual plane that are deleted during the procedure at the last junction $v_{k}$. By assumption and construction, we have $\ell_{-}^{*} \neq r$, and the slope of the segment $\ell_{+}^{*} \ell_{-}^{*} \subset v_{k}^{*}$ connecting their duals in the primal plane (i.e., the slope of $v_{k}^{*}$ ) is smaller than that of the segment $t r$. We claim that 


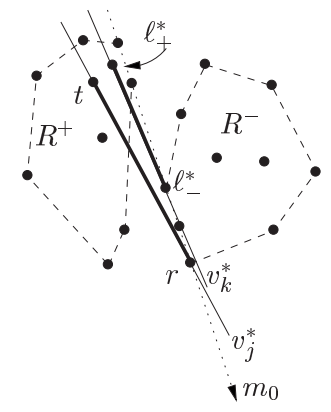

Fig. 25. The segments $\operatorname{tr}$ and $\ell_{+}^{*} \ell_{-}^{*}$ must be convergent.

the two segments $\ell_{+}^{*} \ell_{-}^{*} \subset v_{k}^{*}$ and $\operatorname{tr} \subset v_{j}^{*}$ are convergent. Indeed, since $m_{0}$ (weakly) separates $R^{+}$and $R^{-}$, the closed segment $\ell_{+}^{*} \ell_{-}^{*}$ must meet $m_{0}$, and this must happen at a point to the left of (and above) $r$, or else $r$ would not be an extreme point of $R$ (see Fig. 25). For a similar reason, $\ell_{-}^{*}$ must lie above $v_{j}^{*}$. These facts, together with the slope relationship between $v_{j}^{*}$ and $v_{k}^{*}$, imply that the two segments are convergent. This, in turn, implies that the double wedges dual to $t r$ and to $\ell_{-}^{*}, \ell_{+}^{*}$ are convergent. Since $W\left(v_{k}\right)$ and $W\left(v_{j}\right)$ are contained in these respective double wedges, they are also convergent. However, $W\left(v_{k}\right)$ is bounded by a line of $L_{k}^{+}$and by a line of $L_{k}^{-}$, and Claim 4.1(ii) implies that $v_{j}$ lies between these lines, and hence in $W\left(v_{k}\right)$, showing that $W\left(v_{j}\right)$ and $W\left(v_{k}\right)$ are non-convergent. ${ }^{2}$ This contradiction completes the proof of the claim.

The above argument is valid for any coordinate system whose $y$-axis strictly separates the sets $R^{-}$and $R^{+}$,or, in case $q$ exists, passes through $q$ and strictly separates $R_{0}^{-}$and $R_{0}^{+}$. We specify a coordinate system with this property as follows.

Choose the $y$-axis to be very close to $m_{0}$, so that, in the dual plane, the slope of every line of $L$ passing through $m_{0}^{*}$ has a smaller absolute value than the slope of any other line of $L$; that is, the $x$-coordinates of the points of $m_{0} \cap R$ have smaller absolute values than those of any other point of $R$. See Figs. 26(a), 27(a), and 28(a). In addition, if $q$ exists, we make the $y$-axis pass through $q$, as already stated.

Now we are in a position to define the set of stations $S_{k}$. The reason for choosing the specific way of partitioning $R$, and the coordinate frame, is to force the stations in $S_{k}$ to lie to the left of $v_{1}$, which will be useful when establishing the non-convergence of the segments in $F$ and in $E$. With one possible exception, all stations in $S_{k}$ do indeed lie to the left of $v_{1}$.

Pass to the dual plane. The first junction, $v_{1}$, lies inside or on the boundary of the face $f_{k}$ of $\mathcal{A}\left(L_{k}^{-} \cup L_{k}^{+}\right)$, whose rightmost vertex is $v_{k}$, so that the segment $e=v_{1} v_{k}$ is contained in the closure of $f_{k}$. We distinguish the following cases:

Case A: Suppose first that $v_{k}=m_{0}^{*}$ and that the point c $:=m_{0} \cap m_{1}$ does not belong to $R^{-}$. Let $\ell_{1}^{-}, \ldots, \ell_{v_{1}}^{-}$and $\lambda_{1}^{-}, \ldots, \lambda_{v_{k}}^{-}$denote the lines of $D_{1}^{-}$and all the lines of $L_{k}^{-}=D_{k}^{-}$, respectively, listed in the decreasing order of their slopes. By the special choice of our

\footnotetext{
2 This is a special case of a more general argument, given in Lemma 4.4 below.
} 


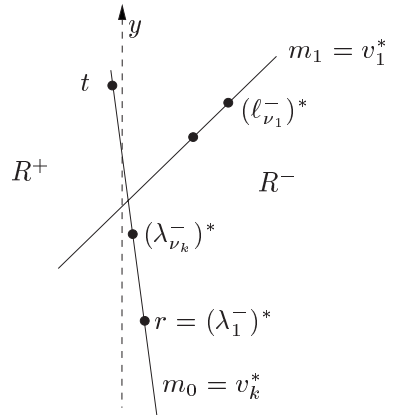

(a)

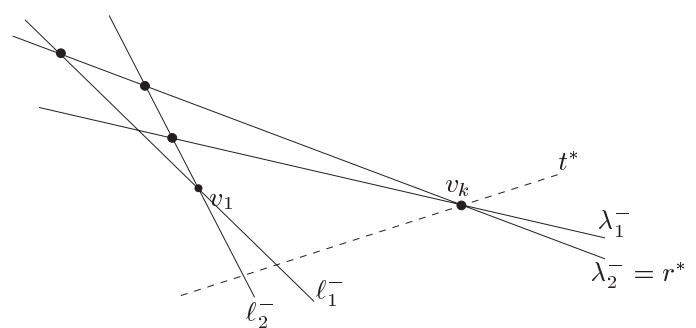

(b)

Fig. 26. Case A of the construction of $S_{k}$, where $v_{k}=m_{0}^{*}$ and $m_{0} \cap m_{1} \notin R^{-}$. (a) The primal structure. (b) The stations in $S_{k}$ (encircled to the left of $v_{1}$ ).

coordinate system, each line $\ell_{i}^{-}$intersects every line $\lambda_{j}^{-}$to the left of $v_{1}$. Indeed, all the lines of $L_{k}^{-}$pass above or through $v_{1}$, by Claim 4.1(ii), but no line passes through both $v_{k}$ and $v_{1}$, because such a line is dual to the point $m_{0} \cap m_{1}$, which we have assumed not to belong to $R^{-}$. The slope of the primal segment $\left(\lambda_{j}^{-}\right)^{*}\left(\ell_{i}^{-}\right)^{*}$ is larger than that of $m_{1}$, because, by what has just been argued, $\left(\lambda_{j}^{-}\right)^{*} \in m_{0}$ lies below $m_{1}$ and to the left of $\left(\ell_{i}^{-}\right)^{*} \in m_{1}$; see Fig. 26(a). Hence the dual intersection point lies to the left of $v_{1}$. Define the last set of stations, $S_{k}$, as the collection of all intersection points of $\ell_{\nu_{1}}^{-}$with the lines $\lambda_{1}^{-}, \ldots, \lambda_{\nu_{k}}^{-}$, and all intersection points of $\lambda_{\nu_{k}}^{-}$with the lines $\ell_{1}^{-}, \ldots, \ell_{\nu_{1}}^{-}$. See Fig. 26(b). Clearly, we have $\left|S_{k}\right|=v_{k}+v_{1}-1$ such stations, all lying to the left of $v_{1}$. Since the total (original) weight of the lines $\ell_{1}^{-}, \ldots, \ell_{v_{1}}^{-}$is at least $d_{1}$, and the total (original) weight of the lines $\lambda_{1}^{-}, \ldots, \lambda_{\nu_{k}}^{-}$is at least $d_{k}$, it follows, as in the construction of the other sets of stations, that the stations in $S_{k}$ generate in this case at least $d_{1}+d_{k}-1$ segments in $E\left(S_{k}\right)$.

Case B: Suppose next that $v_{k}=m_{0}^{*}$ and that the point $c:=m_{0} \cap m_{1}$ does belong to $R^{-}$. Note that if $q$ exists it must coincide with $c$. We first consider the case where $q$ does not exist, and then discuss the modifications that are needed when $q$ exists. The dual line $c^{*}$ passes through both $v_{1}$ and $v_{k}$. Since we assume for now that $q$ does not exist, $c$ does not belong to $R^{+}$. We thus switch to $R^{+}$, and collect stations using the dual lines in $L^{+}$, in a manner similar to that in Case A. All lines in $D_{k}^{+}=L_{k}^{+}$pass strictly below $v_{1}$, and the lines of $D_{1}^{+}$pass strictly above $v_{k}$. Arguing exactly as in Case A, let $\ell_{1}^{+}, \ldots, \ell_{v_{1}}^{+}$and $\lambda_{1}^{+}, \ldots, \lambda_{v_{k}}^{+}$denote the lines of $D_{1}^{+}$and the lines of $D_{k}^{+}=L_{k}^{+}$, respectively, listed in the increasing order of their slopes. The special choice of our coordinate system implies that each line $\ell_{i}^{+}$intersects every line $\lambda_{j}^{+}$to the left of $v_{1}$. Indeed, the slope of the primal segment $\left(\lambda_{j}^{+}\right)^{*}\left(\ell_{i}^{+}\right)^{*}$ is larger than that of $m_{1}$, because $\left(\lambda_{j}^{+}\right)^{*} \in m_{0}$ lies above $m_{1}$ and to the right of $\left(\ell_{i}^{+}\right)^{*} \in m_{1}$. Hence the dual intersection point lies to the left of $v_{1}$. In this case we define $S_{k}$ as the collection of all intersection points of $\ell_{\nu_{1}}^{+}$with the lines $\lambda_{1}^{+}, \ldots, \lambda_{v_{k}}^{+}$, and all intersection points of $\lambda_{v_{k}}^{+}$with the lines $\ell_{1}^{+}, \ldots, \ell_{\nu_{1}}^{+}$. Clearly, we have $\left|S_{k}\right|=v_{k}+v_{1}-1$ such stations, all lying to the left of $v_{1}$, and they generate, as above, at least $d_{1}+d_{k}-1$ segments in $E\left(S_{k}\right)$. 


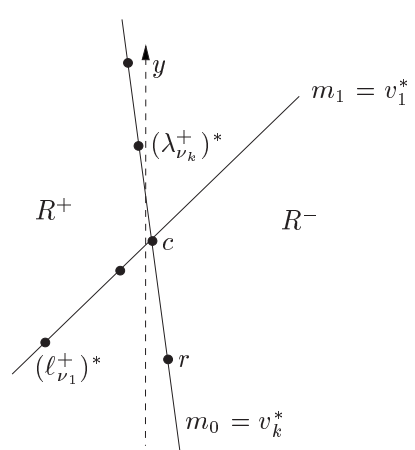

(a)

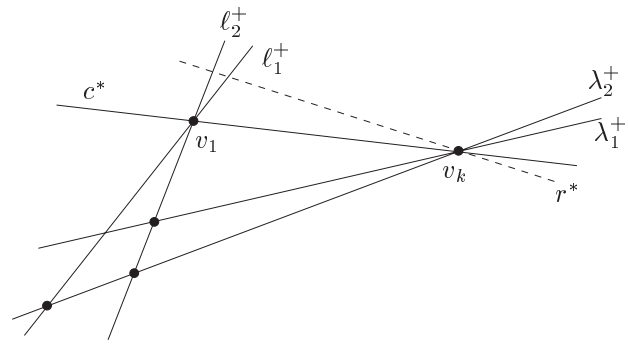

(b)

Fig. 27. Case B of the construction of $S_{k}$, where $v_{k}=m_{0}^{*}$ and $c=m_{0} \cap m_{1} \in R^{-}$. (a) The primal structure. (b) The stations in $S_{k}$ (highlighted to the left of $v_{1}$ ).

Case C: Suppose finally that $v_{k} \neq m_{0}^{*}$. In this case, according to Claim 4.3, $v_{k}$ lies on $r^{*}$ and $v_{k}=1$. Refer to Fig. 28. Again, let $\ell_{1}^{-}, \ldots, \ell_{v_{1}}^{-}$denote the lines of $D_{1}^{-}$, listed in the decreasing order of their slopes. In the dual plane the line $r^{*}$ passes above $v_{1}$ and, by the choice of the coordinate system, it intersects every $\ell_{i}^{-}$to the left of $v_{1}$, with the possible exception of $\ell_{1}^{-}$. The intersection $r^{*} \cap \ell_{1}^{-}$can lie to the right of $v_{1}$ (and of $v_{k}$ ) only if the point $c:=m_{0} \cap m_{1}$ belongs to $R^{-}$and is dual to a line in $D_{1}^{-}$, in which case that line must be $\ell_{1}^{-}=c^{*}$. Note that in this case $r^{*} \cap \ell_{1}^{-}=r^{*} \cap c^{*}$ is identical to the point $m_{0}^{*}$ dual to $m_{0}$, and the choice of the coordinate system implies that this is the rightmost vertex of $\mathcal{A}(L)$ on $r^{*}$. We define $S_{k}$ to be the set of intersection points between the lines $\ell_{1}^{-}, \ldots, \ell_{\nu_{1}}^{-}$and $r^{*}$. Thus, either all points of $S_{k}$, or all but one (namely, $m_{0}^{*}$ ) lie to the left of $v_{1}$. Clearly, we have $\left|S_{k}\right|=v_{1}=v_{k}+v_{1}-1$, and, as above, these stations generate at least $d_{1}+d_{k}-1$ segments in $E$.

We associate with each station $u \in S_{k}$ the double wedge $W(u)$ formed by the two lines $\ell_{i}^{-}, \lambda_{j}^{-}\left(\right.$or $\left.\ell_{i}^{+}, \lambda_{j}^{+}\right)$that meet at $u$.

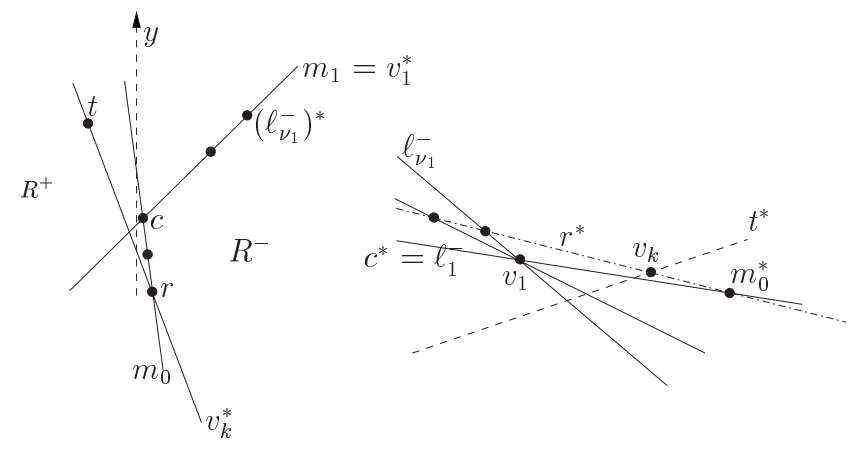

(a)

(b)

Fig. 28. Case $\mathrm{C}$ of the construction of $S_{k}$, where $v_{k} \neq m_{0}^{*}$. (a) The primal structure. (b) The dual picture. 


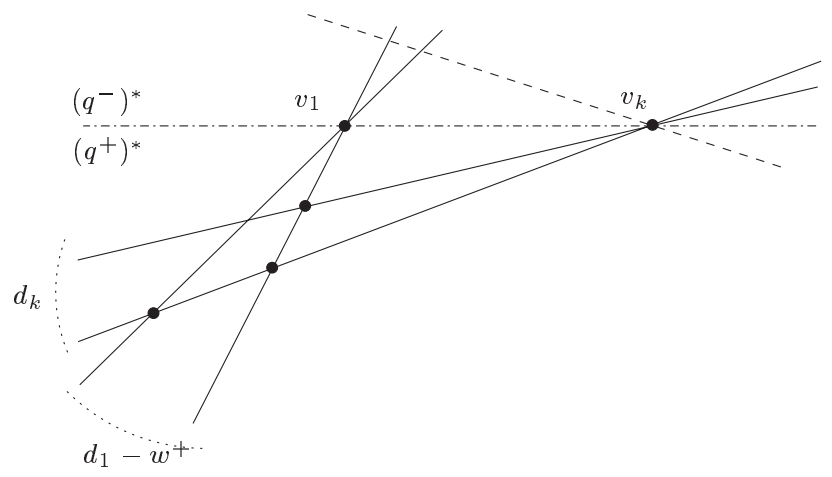

Fig. 29. Constructing $S_{k}$ in the presence of $q^{*}$.

Constructing $S_{k}$ when $q$ Exists. Suppose now that the central bichromatic point $q$ exists. Examining the three cases in the construction of $S_{k}$, we note that Case A cannot arise, because in this case the point $m_{0} \cap m_{1}$, which has to be equal to $q$, does not belong to $R^{-}$, contradicting the definition of $q$. In Case $\mathrm{C}$ we can proceed exactly as above, and collect at least $d_{1}+d_{k}-1$ segments in $E\left(S_{k}\right)$. (In fact, since we use the full weight of $q$, we get $w\left(q^{+}\right)$additional segments in $E\left(S_{k}\right)$.)

It therefore remains to consider Case B, in which $v_{k}=m_{0}^{*}$ and $q=m_{0} \cap m_{1}$. In the dual plane, $q^{*}$ passes through both $v_{1}=m_{1}^{*}$ and $v_{k}=m_{0}^{*}$. Suppose, without loss of generality, that $\left(q^{+}\right)^{*}$ was removed at $v_{1}$. See Fig. 29.

We consider two subcases. In each of them the analysis becomes simpler if $\left(q^{-}\right)^{*} \in$ $D_{k}^{-}$. Moreover, we assume in what follows that $k>2$. The case $k=2$ will be treated separately later.

Case 1: Suppose first that $D_{1}^{+} \backslash\left\{\left(q^{+}\right)^{*}\right\}$ is non-empty. In this case we use the lower grid, to the left of $v_{1}$, formed by the lines of $D_{1}^{+} \backslash\left\{\left(q^{+}\right)^{*}\right\}$ and the lines of $L_{k}^{+}$(which, by assumption, do not include $\left.\left(q^{+}\right)^{*}\right)$. Clearly, $L_{k}^{+}$is not empty. Since $D_{1}^{+}$contains lines other than $\left(q^{+}\right)^{*}$, the lower grid can indeed be used. The lines of $L_{k}^{+}$contribute the full weight $d_{k}$, but the lines of $D_{1}^{+} \backslash\left\{\left(q^{+}\right)^{*}\right\}$ contribute only $d_{1}-w^{+}$overall weight, so the grid generates (at least) $d_{1}+d_{k}-w^{+}-1$ segments in $E\left(S_{k}\right)$.

If $\left(q^{-}\right)^{*} \in D_{k}^{-}$, the loss of $w^{+}$segments in $E\left(S_{k}\right)$, compared with the analysis in the standard case, will be automatically compensated for in the construction of $E\left(v_{k}\right)$, since this analysis gains "for free" the weight $w^{+}$of $\left(q^{+}\right)^{*}$ when it handles the line $\left(q^{-}\right)^{*}$. The case $\left(q^{-}\right)^{*} \notin D_{k}^{-}$will be handled shortly.

Case 2: $\left(q^{+}\right)^{*}$ is the only line in $D_{1}^{+}$. In this case the lower grid does not exist, and we have $d_{1}=w^{+}$. Clearly, there must exist lines of $L_{1}^{-}$through $v_{1}$ other than $\left(q^{-}\right)^{*}$. Moreover, the line $r^{*}$ passes by construction through $v_{k}=m_{0}^{*}$ (see Fig. 27). If $\left(q^{-}\right)^{*} \in L_{k}^{-}$, then $r^{*}$ must also belong to $L_{k}^{-}$, for otherwise it would have to pass through some preceding junction $v_{j}$, so $\left(q^{-}\right)^{*}$ would pass below $v_{j}$, which is impossible for lines of $L_{k}^{-}$. If $\left(q^{-}\right)^{*} \notin L_{k}^{-}$, then all the lines of $L_{k}^{-}$(which is a non-empty set) pass above $v_{1}$. Hence we may use in this case the upper grid, which generates at least $d_{1}-w_{1}^{-}+d_{k}-w_{k}^{-}-1$ segments in $E\left(S_{k}\right)$, where $w_{1}^{-}, w_{k}^{-}$are the weights that $\left(q^{-}\right)^{*}$ contributes at the respective junctions $v_{1}, v_{k}$. 
If $w_{1}^{-}=w_{k}^{-}=0$, we obtain the standard bound $d_{1}+d_{k}-1$.

If $w_{1}^{-}>0$ and $w_{k}^{-}=0$, we are in a symmetric version of the situation in Case 1 that still needs to be treated. Both versions will be treated together below.

If $w_{1}^{-}=0$ and $w_{k}^{-}>0$, we automatically compensate for the loss of $w_{k}^{-}$segments in the count, in the construction of $E\left(v_{1}\right)$, which, similar to the argument in Case 1, gives us $w\left(q^{-}\right) \geq w_{k}^{-}$extra segments "for free".

If $w_{1}^{-}>0$ and $w_{k}^{-}>0$, we interpret the bound in the context of Claim 4.2, except that our bound is one smaller than what the claim guarantees.

It remains to analyze the subcases where $\left(q^{-}\right)^{*} \notin D_{k}^{-}$, and where we still need to compensate for the loss of $\max \left\{w^{+}, w_{1}^{-}\right\}$segments in $E\left(S_{k}\right)$.

Note that this loss is identical to the potential loss at $E\left(S_{1}\right)$, discussed above. We compensate for it in the same way-by including $q$ in the construction of $E\left(v_{k}\right)$. The same analysis shows that we can always compensate for the loss, unless $\min \left\{w^{+}, w^{-}\right\}=1$, in which case we lose one segment in the count.

For this analysis to work, it is crucial that $k>2$. Otherwise we need to compensate twice for the loss of $\max \left\{w^{+}, w_{1}^{-}\right\}$segments, once in $E\left(S_{1}\right)$ and once in $E\left(S_{k}\right)$, but if $v_{2}=v_{k}$ we can compensate for it only once.

As in the case of $S_{1}$, here too we pay the price of replacing $W\left(v_{k}\right)$ by the narrower double wedge bounded by $q^{*}$ and by the shallowest line of the set among $D_{k}^{-}, D_{k}^{+}$to which $q^{*}$ was not adjoined. Nevertheless, we show in Section 4.5 that this does not affect the pairwise non-convergence of the segments in $F$.

\subsection{Counting the Number of Segments in E}

The Standard Case. We first consider the standard case, where $q$ does not exist. Combining the contributions in (2) and (3) with the contribution in (1), we obtain that $E$ consists of a total of at least

$$
k+t+\sum_{j=1}^{t} \kappa_{j}\left(w_{j}-1\right)+2 \sum_{i=1}^{k} d_{i}-\sum_{i \in I_{1}}\left(a_{i}+a_{i+1}\right)-\sum_{i \in I_{2}} 1
$$

segments, where $I_{1}$ is the set of indices $i$ for which there exists a (unique) line which contributes to both $d_{i}$ and $d_{i+1}$ (or to $d_{1}$ and $d_{k}$, for $i=k$ ), and $I_{2}$ is the complementary set.

Assume first that there is no line that contributes to all the $k$ weights $d_{1}, \ldots, d_{k}$. Then each line $\ell_{j}$ can contribute to at most $\kappa_{j}-1$ pairs of successive weights $d_{i}, d_{i+1}$, and each of the corresponding terms $\left(a_{i}+a_{i+1}\right)$ is at most $w_{j}$. Even if there exists a line that contributes to all $k$ weights $d_{i}$, it does not affect the construction of the segments from the stations of $S_{k}$, which always produces at least $d_{1}+d_{k}-1$ segments (when $q$ does not exist). That is, we can always pretend that $k \in I_{2}$, so the analysis proceeds in the same way in this case, too. The remaining pairs of successive weights contribute -1 to the expression above (in the summation over $i \in I_{2}$ ). Therefore, an overestimate of the (absolute value of the) negative terms in (4) is $\sum_{j=1}^{t}\left(\kappa_{j}-1\right) w_{j}+\left(k-\sum_{j=1}^{t}\left(\kappa_{j}-1\right)\right)$. 
Using the fact that $2 \sum_{i=1}^{k} d_{i}=\sum_{j=1}^{t} w_{j}=n-1$, the bound in (4) is greater than or equal to

$$
k+t+\sum_{j=1}^{t} \kappa_{j}\left(w_{j}-1\right)+\sum_{j=1}^{t} w_{j}-k-\sum_{j=1}^{t}\left(\kappa_{j}-1\right)\left(w_{j}-1\right)=2 \sum_{j=1}^{t} w_{j}=2 n-2 .
$$

The Case where q Exists. The differences between this case and the standard case are:

(i) We may lose one segment in $E\left(v_{1}\right)$.

(ii) We may lose one segment in $E\left(S_{1}\right)$. Even if we do not lose the segment, we may collect there only $d_{1}+d_{2}-\left(a_{1}+a_{2}\right)$ segments, where one of $a_{1}, a_{2}$ is 0 .

(iii) A similar situation may occur for $S_{k}$.

(iv) It is possible that $\left(q^{-}\right)^{*}$ or $\left(q^{+}\right)^{*}$ contributes weight to all junctions $v_{1}, \ldots, v_{k}$, which may cause the set $I_{1}$ to consist of all indices $1, \ldots, k$, and $I_{2}$ to be empty.

Assume first that the situation in (iv) does not arise. Then the analysis proceeds as in the standard case, since, as is easily verified, it is not affected by having some of the $a_{i}$ 's vanish, except that we need to subtract 3 from the overall count, to accommodate the potential losses in (i)-(iii). Hence, in this case we have $|E| \geq 2 n-5$.

If the situation in (iv) arises, say, with $\left(q^{-}\right)^{*}$ being the line that contributes weight to all junctions, then $\sum_{i \in I_{1}}\left(a_{i}+a_{i+1}\right)=2 w^{-}$, and all lines $\ell_{j} \neq\left(q^{-}\right)^{*}$ have $\kappa_{j}=1$. The total number of segments in $E$ is therefore at least (without loss of generality, we assume that $\left(q^{-}\right)^{*}$ is the $t$ th line)

$$
\begin{aligned}
k\left(w^{-}-1\right) & +\sum_{j=1}^{t-1}\left(w_{j}-1\right)+n-1-2 w^{-}+k+t-3 \\
& =(k-2) w^{-}+(n-1)+t-(t-1)+\left(n-1-w^{-}\right)-3 \\
& =(k-3) w^{-}+2 n-4 .
\end{aligned}
$$

Hence, if $k \geq 3$, we have $|E| \geq 2 n-4$. (Recall that the count so far actually relied on the assumption that $k \geq 3$.)

It thus remains to consider the case where only two junctions are generated. In this case, by construction, all the lines of $L$ must pass either through $v_{1}$ or through $v_{2}$; see Fig. 30(a). Hence, in the primal plane, all points of $R$ must lie on the lines $m_{0}$ and $m_{1}$, with $q$ lying on both lines; see Fig. 30(b).

In this very degenerate case, we construct $E$ explicitly, working in the primal plane $\pi$, as follows. Denote by $W_{1}^{+}, W_{1}^{-}, W_{2}^{+}$, and $W_{2}^{-}$the overall weight of all points of $R^{+} \cap m_{1}, R^{-} \cap m_{1}, R^{+} \cap m_{0}$, and $R^{-} \cap m_{0}$, respectively, excluding $q$ in all four cases.

(i) Apply Theorem 3.1 or Corollary 3.2 in the planes defined by $m_{0}$ and $m_{1}$, respectively, and by $p_{0}$ (or, equivalently, at the dual junctions $m_{1}^{*}=v_{1}$ and $m_{0}^{*}=v_{2}$ ). To be on the safe side, we assume that only Corollary 3.2 can be used at either junction, and, as usual, we subtract 1 from the bound at $v_{1}$ to allow for potential double counting of a segment. This yields a total of at least

$$
\begin{aligned}
\left(W_{1}^{+}+W_{1}^{-}\right. & +w(q)-1)+\left(W_{2}^{+}+W_{2}^{-}+w(q)\right) \\
& =\left(W_{1}^{+}+W_{1}^{-}+W_{2}^{+}+W_{2}^{-}+w(q)\right)+w(q)-1 \\
& =(n-1)+w(q)-1
\end{aligned}
$$

segments in $E$. 

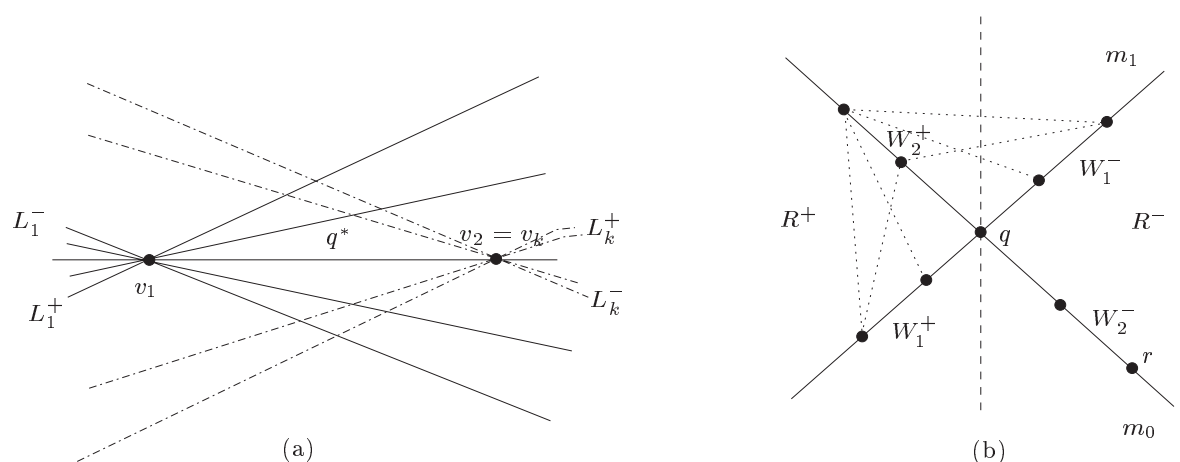

Fig. 30. The case of only two junctions in the presence of $q^{*}$ : (a) the dual configuration, and (b) the primal configuration (in $\pi$ ).

(ii) Suppose, without loss of generality, that $W_{2}^{-} \leq W_{2}^{+}$. We then generate segments in $F$, in addition to those lying on $m_{0}, m_{1}$, as shown in Fig. 30(b). That is, we connect the point of $R^{+} \cap m_{0}$ farthest from $q$ to all the points on $m_{1}$, excluding $q$, and connect the two points of $R^{+} \cap m_{1}, R^{-} \cap m_{1}$ farthest from $q$ to all the points of $R^{+} \cap m_{0}$. Here it is easy to verify directly that all these segments, including the segments $f\left(v_{1}\right) \subset m_{1}, f\left(v_{2}\right) \subset m_{0}$ (where the first may degenerate to the singleton point $q$, but is still considered to lie along $m_{1}$ ), are pairwise non-convergent. The $F$-segments that we have constructed are dual to the stations in $S_{1} \cup S_{2}$. The total number of segments in $E$ that are generated from these stations, in the standard manner, is at least

$\left(W_{1}^{-}+W_{2}^{+}-1\right)+\left(W_{1}^{+}+W_{2}^{+}-1\right) \geq W_{1}^{+}+W_{1}^{-}+W_{2}^{+}+W_{2}^{-}-2=n-1-w(q)-2$.

Adding the bounds from (i) and (ii), we get $|E| \geq 2 n-5$. We note that the configuration in Fig. 31 falls into this case.

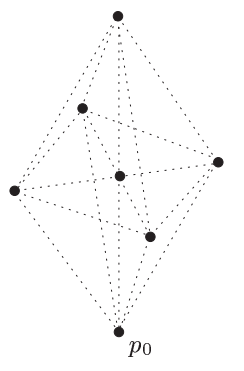

(i)

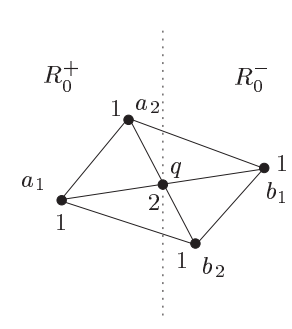

(ii)

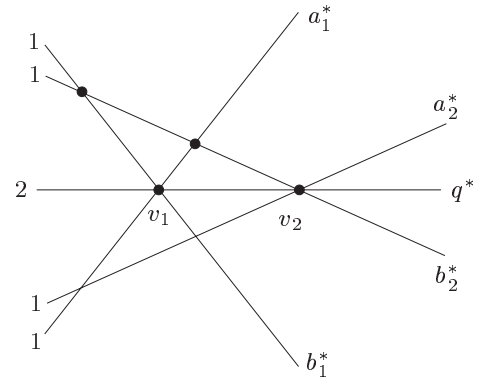

(iii)

Fig. 31. (i) The set of Fig. 1 with $n=7$ points that determines $2 n-5=9$ directions. (ii) The weighted set $R$ in the primal plane $\pi$, obtained by projecting from $p_{0}$, with the central bichromatic point $q$ of weight 2 . (iii) The dual construction of junctions and stations. We collect four segments in $E\left(v_{1}\right)$ (using Corollary 3.2), three in $E\left(v_{2}\right)$ (using Corollary 3.2 and subtracting 1), one in $E\left(S_{1}\right)$, and one in $E\left(S_{2}\right)$, for a total of nine pairwise non-convergent segments. 


\subsection{Pairwise Non-Convergence of the Collected Segments}

To complete the proof, we have to show that no pair of segments in $E$ are convergent. We first show:

Lemma 4.4. Let $Q$ denote the set of all junctions and stations that we have collected. For any $u, v \in Q$, the segments $f(u)$ and $f(v)$ associated with these vertices are nonconvergent in the primal plane $\pi$.

Proof. We first consider the standard case, where $q$ does not exist. Let $u, v \in Q$ with $u$ lying to the left of $v$. The property that $f(u)$ and $f(v)$ are non-convergent is dual to the property that $W(u)$ and $W(v)$ are non-convergent, that is, either $u$ lies in (the closure of) $W(v)$ or $v$ lies in (the closure of) $W(u)$. We distinguish between several cases:

Case A: Both $u$ and $v$ are junctions. Put $u=v_{i}$ and $v=v_{j}$, with $i<j$. Then $W(v)$ is bounded by a line $\ell \in L_{j}^{+}$and by a line $\ell^{\prime} \in L_{j}^{-}$. By Claim 4.1(ii), $u=v_{i}$ lies between these two lines, and thus belongs to $W\left(v_{j}\right)=W(v)$.

Case B: $u$ is a junction and $v$ is a station not in $S_{k}$. Put $u=v_{i}$ and let $S_{j}$ be the set of stations that contains $v$, where $i \leq j<k$. Then $W(v)$ is bounded by two lines $\ell, \ell^{\prime}$, where either $\ell \in L_{j}^{+}$and $\ell^{\prime} \in L_{j+1}^{-}$, or $\ell \in L_{j+1}^{+}$and $\ell^{\prime} \in L_{j}^{-}$. By construction, we have in both cases $\ell \in L_{j}^{+}$and $\ell^{\prime} \in L_{j}^{-}$, and the analysis is completed as in Case A.

Case $C: u$ is a station not in $S_{k}$ and $v$ is a junction or a station not in $S_{k}$. Let $S_{i}$ be the set of stations containing $u$. The arguments in Cases A and B imply that $v_{i} \in W(v)$. If $v$ is also a station in $S_{i}$ or $v=v_{i+1}$ then it is clear from the construction of $S_{i}$ that $W(u)$ and $W(v)$ are non-convergent (see Fig. 19). Suppose then that $v$ lies to the right of $v_{i+1}$. Then both $v_{i}$ and $v_{i+1}$ lie in the left wedge of $W(v)$, and $u$ is incident to a line $\ell$ that passes through $v_{i}$ and to a line $\ell^{\prime}$ that passes through $v_{i+1}$. If $u \notin W(v)$ then a boundary line of $W(v)$ must separate $u$ from $v_{i}$ and $v_{i+1}$, in which case $v \in W(u)$; compare with Fig. 14(a).

Case D: $u$ is a station in $S_{k}$ to the left of $v_{1}$ and $v$ is a junction or station. If both $u$ and $v$ belong to $S_{k}$, then the claim follows easily from the construction of $S_{k}$. We thus suppose that $v \notin S_{k}$. Then we have $v \in\left\{v_{i}\right\} \cup S_{i} \cup\left\{v_{i+1}\right\}$, for some $1 \leq i<k$.

We start with the case $v_{k}=m_{0}^{*}$. Refer to Fig. 32. Suppose that $u \in S_{k}$ is the intersection point of two lines $\ell$ and $\lambda$, passing through $v_{1}$ and $v_{k}$, respectively, which, without loss of generality, we assume to belong to $L^{-}$. If $v$ is contained in the double wedge bounded by $\ell$ and $\lambda$, then $v \in W(u)$, so $W(v)$ and $W(u)$ are non-convergent. Otherwise, since $u$ lies to the left of $v_{1}, v$ lies either above $\lambda$ or below $\ell$. If $v$ is above $\lambda$, then it is not a junction, so it must be the crossing point of a line $\ell^{+} \in D_{i}^{+}$and a line $\ell^{-} \in D_{i+1}^{-}$. See Fig. 32(a). Both $v_{i}$ and $v_{i+1}$ lie on or below $\lambda$, so the left portion of the double wedge bounded by $\ell^{-}$and $\ell^{+}$contains $u$. Thus, we have $u \in W(v)$. If, on the other hand, $v$ is below $\ell$, as in Fig. 32(b), then it is either a junction or a station, and it is the crossing point of a line $\ell^{-} \in L^{-}$and a line $\ell^{+} \in L^{+}$which bound $W(v)$, such that either both $\ell^{+}$and $\ell^{-}$are in $D_{i}$ (if $v=v_{i}$ is a junction), or $\ell^{-} \in D_{i}^{-}$and $\ell^{+} \in D_{i+1}^{+}$(if $v \in S_{i}$ is a station). Now $\ell^{-}$must pass above (or through) $v_{1}$ and hence above $u$, while $\ell^{+}$must pass below $u$. Again we can conclude that the left portion of the double wedge bounded 


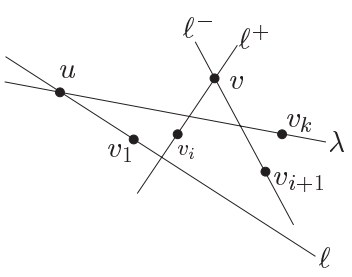

(a)

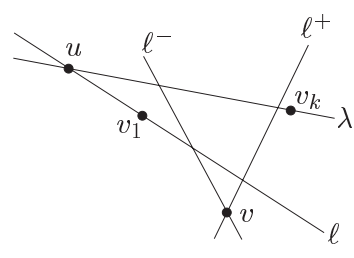

(b)

Fig. 32. The proof that $W(u)$ and $W(v)$ are non-convergent when $u$ is a station to the left of $v_{1}$. (a) $v$ lies above $W(u)$. (b) $v$ lies below $W(u)$.

by $\ell^{-}$and $\ell^{+}$, and thus $W(v)$, contains $u$. The case where $u$ lies on two lines of $L^{+}$is handled in a fully symmetric manner.

If $v_{k} \neq m_{0}^{*}$, the above argument can be repeated verbatim for stations $u \in S_{k}$ to the left of $v_{1}$. If $v=m_{0}^{*}$, the sole station to the right of $v_{k}$, the claim is immediate from the construction of $S_{k}$. Hence, Case D of the claim holds in either case.

Case E: $u$ is a junction or station not in $S_{k}$ and $v$ is a station in $S_{k}$ (to the right of $v_{k}$ ). Case E can arise only when $v=m_{0}^{*} \in S_{k}$. Now it is simplest to establish the claim in the primal plane, by noting that the segment dual to $W(v)$ lies on the line $m_{0}$, and that, by construction (since $u \notin S_{k}$ ), the segment dual to $W(u)$ must connect a point of $R^{-}$to a point of $R^{+}$, and thus must intersect $m_{0}$, showing that these two segments are non-convergent.

Consider next the case where the central bichromatic point $q$ exists, which requires a few modifications in the preceding analysis. First, if both $\left(q^{-}\right)^{*}$ and $\left(q^{+}\right)^{*}$ belong to $D_{1}$, the double wedge $W\left(v_{1}\right)$ degenerates to the single line $q^{*}$. (We still consider it to have $v_{1}$ as an apex. In the primal plane the segment $f\left(v_{1}\right)$ degenerates to the singleton point $q$, but it is still considered to lie along the line $v_{1}^{*}$.) It is easily verified, though, by specializing Cases A, B, D, and $\mathrm{E}$ to this configuration, that $W\left(v_{1}\right)$ and any other wedge $W(v)$ in our collection are still non-convergent.

The presence of $q$ does not affect any other case in the preceding analysis, as long as we were not forced to include $q$ in the construction of $E\left(v_{2}\right)$ or $E\left(v_{k}\right)$. Suppose then that we had to include $q$ in the construction of $E\left(v_{2}\right)$ (even though neither $\left(q^{+}\right)^{*}$ nor $\left(q^{-}\right)^{*}$ belonged to $\left.D_{2}\right)$. In Case A, $v_{2}$ is contained in $W\left(v_{j}\right)$ for any $j>2$ (the case where $W\left(v_{k}\right)$ was also shrunk will be treated below), so it only remains to consider the case $u=v_{1}, v=v_{2}$, which still works, since $v_{1} \in q^{*}$, and thus $v_{1}$ still lies in the modified $W\left(v_{2}\right)$. Case B is not affected by the shrinking of $W\left(v_{2}\right)$. In Case C we only need to consider the subcase when $u \in S_{1}$, and the property continues to hold since $v_{2} \in W(u)$. In Case D we have $v_{2} \in W(u)$, which easily follows from the fact that $v_{2} \in q^{*}$; see Fig. 33. Case $\mathrm{E}$ is argued as in the standard case.

Suppose finally that we had to include $q$ in the construction of $E\left(v_{k}\right)$ (even though neither $\left(q^{+}\right)^{*}$ nor $\left(q^{-}\right)^{*}$ belonged to $\left.D_{k}\right)$. Recall that this can arise only in Case B of the construction of $S_{k}$, where $v_{k}=m_{0}^{*}$. Now, except for the stations in $S_{k}$, for any other vertex $u \in Q, f(u)$ connects a point of $R^{+}$and a point of $R^{-}$, and thus the line containing $f\left(v_{k}\right)$, namely $m_{0}$, must cross $f(u)$, so $f(u)$ and $f(v)$ are non-convergent. If $u$ is a station in $S_{k}$, then $v_{k} \in W(u)$, by construction.

Hence the lemma also holds when $q$ exists. 


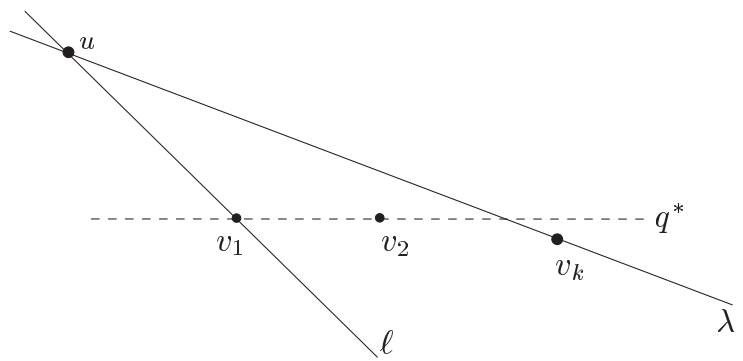

Fig. 33. Case $\mathrm{D}$ of the analysis when $W\left(v_{2}\right)$ is shrunk: $v_{2} \in q^{*}$ lies above $\ell$ and below $\lambda$ and thus $v_{2} \in W(u)$.

Non-Convergence of the Elements of $E$. Recall that, for each $v \in Q$, the points of $P$ that span the segments in $E(v)$ are those points that project to the line containing $f(v)$ in $\pi$, so that their projections are dual to lines in $L$ that either were removed at $v$ or had their weights reduced there (if $v$ is a junction), or are the two lines incident to $v$ (if $v$ is a station).

Moreover, each segment $e$ in $E(v)$ has the property that either its projection on $\pi$ contains the segment $f(v)$ or it is a point not in the interior of $f(v)$; the latter case arises when $e$ is contained in a ray emanating from $p_{0}$, a situation that can arise when we apply Theorem 3.1 or Corollary 3.2 at one of the junctions $v_{1}, \ldots, v_{k}$.

Let $e_{1}$ and $e_{2}$ be two segments in $E$. For $i=1,2$, let $u_{i}$ denote the vertex in $Q$ for which $e_{i} \in E\left(u_{i}\right)$, and set $f_{i}=f\left(u_{i}\right)$. It suffices to consider the case $u_{1} \neq u_{2}$.

The segments $f_{1}$ and $f_{2}$ are non-convergent in $\pi$. If the projections $\bar{e}_{1}$ and $\bar{e}_{2}$ on $\pi$ from $p_{0}$ of $e_{1}$ and $e_{2}$, respectively, are segments (so that they contain $f_{1}$ and $f_{2}$, respectively), then $\bar{e}_{1}$ and $\bar{e}_{2}$ are non-convergent in $\pi$, which is easily seen to imply that $e_{1}$ and $e_{2}$ are non-convergent in $\mathbb{R}^{3}$. If the projections of both $e_{1}$ and $e_{2}$ are points on $\pi$, then $e_{1}$ and $e_{2}$ share $p_{0}$ as an endpoint and therefore are non-convergent.

We are left with the case in which, without loss of generality, $e_{1}$ projects from $p_{0}$ to a point $x \in \pi$ (which is on the line containing $f_{1}$ but not in the interior of $f_{1}$ ), whereas $e_{2}$ projects to a segment $e_{2}^{\prime}$ containing $f_{2}$. See Fig. 34. The point $x$ may be assumed to lie on the line containing $f_{2}$, for otherwise $e_{1}$ and $e_{2}$ are noncoplanar, and therefore

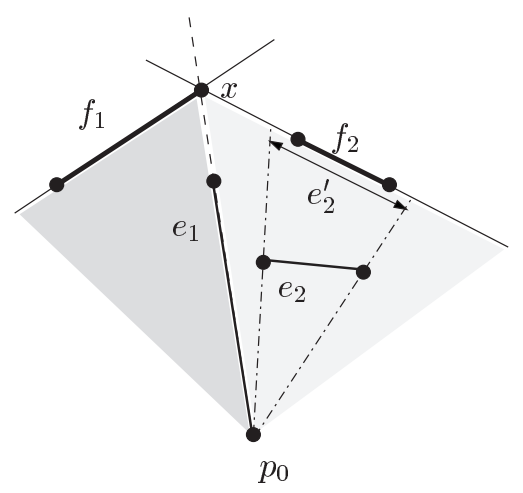

Fig. 34. Non-convergence of $e_{1}$ and $e_{2}$ : an impossible configuration. 


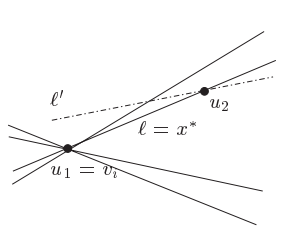

(a)

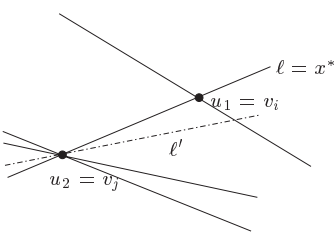

(b)

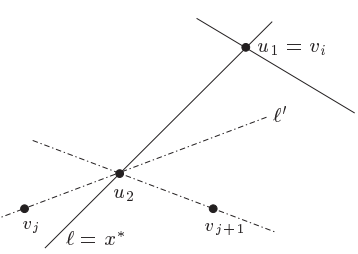

(c)

Fig. 35. Showing the impossibility of the configuration in Figure 34. (a) $u_{1}$ is to the left of $u_{2}$. (b) $u_{2}$ is a junction to the left of $u_{1}$. (c) $u_{2}$ is a station to the left of $u_{1}$.

non-convergent. If $x \in e_{2}^{\prime}$ then clearly $e_{1}$ and $e_{2}$ are non-convergent, so we may assume that $x \notin e_{2}^{\prime}$. It follows that $x \notin f_{2}$ and since $f_{1}$ and $f_{2}$ are non-convergent, $x$ must be an endpoint of $f_{1}$ (otherwise $f_{1}$ and $f_{2}$ would be convergent, because the lines on $\pi$ that contain them meet at $x$ which lies outside both segments).

We claim that this case is impossible. Indeed, we have already noted that if $x$ is an endpoint of $f_{1}$, then the vertex $u_{1}$ dual to $f_{1}$ must be a junction $v_{i}$. The line $\ell$ dual to $x$ passes through both vertices $u_{1}=v_{i}$ and $u_{2}$, and it is the shallowest line in either $D_{i}^{+}$or $D_{i}^{-}$. If the central bichromatic point $q$ exists, and $u_{2}$ is not a station in $S_{k}$, then $\ell$ cannot be equal to $q^{*}$, i.e., $x$ cannot be equal to $q$, because, by construction, $q$ must lie inside $f_{2}$, which is delimited by a point of $R^{+}$and a point of $R^{-}$. Hence, we may assume, without loss of generality, that $\ell \in L^{+} \backslash\left\{\left(q^{+}\right)^{*}\right\}$. The case where $q$ exists and $u_{2}$ is a station in $S_{k}$ will be considered later.

Case 1: $u_{1}=v_{i}$ lies to the left of $u_{2}$. Since $x$ lies outside $f_{2}$, the endpoint of $f_{2}$ nearer to $x$ is dual to a line $\ell^{\prime} \in L_{i}^{+}$that passes through $u_{2}$ and has smaller slope than that of $\ell$. (Since $u_{2}$ is constructed after $u_{1}$, the lines that define $W\left(u_{2}\right)$ must belong to $L_{i}$.) However, then $\ell^{\prime}$ must pass above $v_{i}$ which is a contradiction since all the lines in $L_{i}^{+}$ must pass through or below $v_{i}$. See Fig. 35(a).

Case 2: $u_{1}=v_{i}$ lies to the right of $u_{2}$. Assume first that $u_{2}$ is not a station in $S_{k}$. Let $1 \leq j<i$ be the index such that either $u_{2}=v_{j}$ or $u_{2}$ is a station in $S_{j}$. Since $x$ lies outside $f_{2}$, the $R^{+}$-endpoint of $f_{2}$ (the one nearer to $x$ ) is dual to a line $\ell^{\prime} \in D_{j}^{+} \cup D_{j+1}^{+}$, which is shallower than $\ell$ (since $x$ lies outside $f_{2}$ ). If $u_{2}=v_{j}$ then $\ell^{\prime} \in D_{j}^{+}$, and, by construction, $\ell$ must have also been removed at $v_{j}$ or at an earlier junction, and thus it cannot be dual to an endpoint of $f_{1}$ (because such a point must be the dual of some line in $L_{i}^{+}$). See Fig. 35(b). Hence this case is impossible. Suppose then that $u_{2}$ is a station in $S_{j}$. Regardless of whether $\ell^{\prime} \in D_{j}^{+}$or $\ell^{\prime} \in D_{j+1}^{+}$, since $\ell$ is steeper than $\ell^{\prime}, v_{j+1}$ lies below $\ell$. Hence, we must have $i>j+1$, and, since $\ell \in L_{i}^{+}$, we obtain a contradiction to Claim 4.1(ii); see Fig. 35(c).

Finally, assume that $u_{2}$ is a station in $S_{k}$ to the left of $v_{1}$. Suppose first that $u_{2}$ lies on a line $\ell^{-} \in D_{1}^{-}$and a line $\lambda^{-} \in D_{k}^{-}=L_{k}^{-}$. In this case, $\ell^{\prime}=\lambda^{-}$and $\ell$ passes above $v_{1}$, which is impossible, since $\ell \in L_{1}^{+}$; see Fig. 36(a). Suppose next that $u_{2}$ lies on a line $\ell^{+} \in D_{1}^{+}$and a line $\lambda^{+} \in D_{k}^{+}=L_{k}^{+}$. Since $x$ lies outside $f_{2}, \ell$ is not contained in $W\left(u_{2}\right)$. If $\ell$ is shallower than $\lambda^{+}$(see Fig. 36(b)), then $\ell^{\prime}=\lambda^{+}$, and $v_{i}$ lies below $\lambda^{+} \in L_{k}^{+}$, which is impossible. If $\ell$ is steeper than $\ell^{+}$(see Fig. 36(c)), then $\ell^{\prime}=\ell^{+}$, and $v_{1}$ lies below $\ell$, which is impossible, since $\ell \in L_{1}^{+}$. (Note that in all three cases, $\ell$ cannot be equal to $q^{*}$, because $q^{*}$ passes through $v_{1}$ and $\ell$ does not.) 


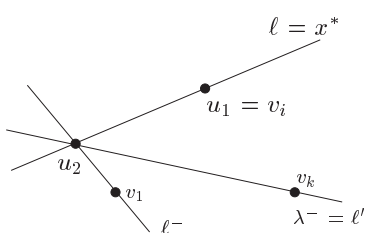

(a)

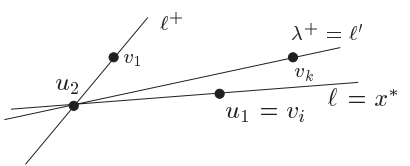

(b)

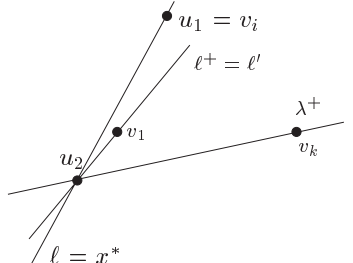

(c)

Fig. 36. Showing the impossibility of the configuration in Figure 34 when $u_{2}$ is a station of $S_{k}$ to the left of $v_{1}$, and $\ell \in L^{+}$. (a) $u_{2}$ is formed by two lines of $L^{-}$. (b) $u_{2}$ is formed by two lines of $L^{+}$, and $\ell$ is shallower than both of these lines. (c) $u_{2}$ is formed by two lines of $L^{+}$, and $\ell$ is steeper than both of these lines.

All these contradictions show that Case 2 is also impossible.

This establishes the non-convergence of the segments in $E$, and thus, at long last, completes the proof of Theorem 1.3.

As mentioned earlier, we can get a better bound when $q$ does not exist:

Theorem 4.5. Let $P$ be a set of $n \geq 6$ noncoplanar points in $\mathbb{R}^{3}$, such that $n$ is odd, and there exists an extreme point $p_{0}$ of $P$, such that the projection of $P$ from $p_{0}$ produces a set $R$ without a central bichromatic point. Then $P$ determines at least $2 n-2$ segments, no two of which are convergent.

This strengthens the bound $2 n-3$ in Conjecture 1 of Blokhuis and Seress [2] for $n$ odd.

\section{Extensions and Open Problems}

In this section we consider several extensions of our results, prove some of them, and leave the others as open problems.

The most obvious open problem is to obtain the exact worst-case bound for $n$ even. Currently there is a small gap between our lower bound $2 n-7$ and the best known construction, which gives $2 n-3$ pairwise non-convergent segments.

Theorem 1.3 yields the following extension to four dimensions. It settles Conjecture 9 of Blokhuis and Seress [2] in the affirmative for $d=4$ and for even $n$.

Theorem 5.1. Let $P$ be a set of $n$ points in $\mathbb{R}^{4}$, not contained in a hyperplane and not having three collinear points. Then $P$ determines at least $3 n-8$ different directions, if $n$ is even, and at least $3 n-10$ different directions if $n$ is odd. The bound is sharp for every even $n \geq 8$.

Proof. Let $p_{0}$ be the lowest point of $P$ (in the $x_{4}$-direction). Let $H$ be a horizontal hyperplane (parallel to the $x_{1} x_{2} x_{3}$-space) far above all the points of $P$. Applying a small rotation to $P$, we may assume that $H$ is not parallel to any segment determined by $P$. 


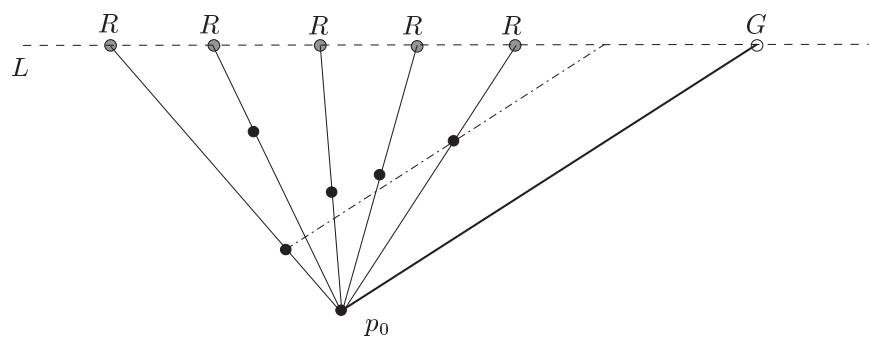

Fig. 37. The green point determined by a red segment in $H$.

Project the points of $P \backslash\left\{p_{0}\right\}$ centrally from $p_{0}$ onto $H$, and color the projected images red. For each direction $\gamma$ determined by $P$, let $L_{\gamma}$ denote the line parallel to $\gamma$ and passing through $p_{0}$. If a direction $\gamma$, determined by $P$, is not obtained through $p_{0}$, let $b_{\gamma}$ denote the intersection point of $L_{\gamma}$ with $H$. Color all such points $b_{\gamma}$ green. Clearly, every red or green point on $H$ gives rise to a different direction determined by $P$, and all these points are distinct. The number of red points on $H$ is $n-1$.

Since $P$ is not contained in a hyperplane, the red points on $H$ are not coplanar. Therefore, by Theorem 1.3, they determine at least $2(n-1)-5=2 n-7$ pairwise non-convergent segments, if $n-1$ is odd, and at least $2(n-1)-7=2 n-9$ pairwise non-convergent segments, if $n-1$ is even.

We claim that along each line $L$ in $H$ passing through two or more red points there is a green point that lies outside the convex hull of the red points on $L$. Indeed, consider the 2-plane through $p_{0}$ and $L$. The direction $\gamma$ in 4-space, determined by the two points of $P$ that project to the two extreme red points on $L$, is not obtained through $p_{0}$, and thus yields the desired green point outside the convex hull of the red points on L. See Fig. 37 . Therefore, every collection of $m$ pairwise non-convergent segments determined by the red points on $H$ gives rise to $m$ distinct green points on $H$, formed in the manner just described. No two such green points can coincide, for that would make the corresponding red segments convergent.

It follows that the number of points on $H$ is at least $n-1+2 n-7=3 n-8$, if $n$ is even, and at least $n-1+2 n-9=3 n-10$, if $n$ is odd.

We next show that the bound is sharp for even $n \geq 8$. The construction extends the one depicted in Fig. 1. Specifically, let $P$ be the set of the vertices of a regular $(n-4)$-gon $Q$ in the $x_{1} x_{2}$-plane, centered at the origin, and of the four points $\pm e_{3}=(0,0, \pm 1,0)$, $\pm e_{4}=(0,0,0, \pm 1)$. It is easy to see that $P$ determines exactly $3 n-8$ different directions: $n-4$ directions in the $x_{1} x_{2}$-plane, $n-4$ directions obtained by connecting the vertices of $Q$ to $e_{3}, n-4$ directions obtained by connecting the vertices of $Q$ to $e_{4}$, and four directions determined by $\pm e_{3}, \pm e_{4}$.

A major generalization of Theorem 5.1, still in four dimensions, would be to establish the following conjecture:

Conjecture A. Any set $P$ of $n$ points in $\mathbb{R}^{4}$, not contained in a single hyperplane, determines at least $3 n-c$ pairwise non-convergent segments, for some constant $c$ (that might be larger than those in the theorem). 
This conjecture would imply, by an appropriate extension of the preceding proof, that any set of $n$ points in $\mathbb{R}^{5}$, not contained in a hyperplane, and not having three collinear points, determines at least $4 n-(c+4)$ different directions.

The final grand challenge is to establish the following conjecture, which strengthens Conjecture 9 of Blokhuis and Seress [2]:

Conjecture B. Any set $P$ of $n$ points in $\mathbb{R}^{d}$, for $d \geq 4$, not contained in a single hyperplane, determines at least $(d-1) n-c_{d}$ pairwise non-convergent segments, for some constant $c_{d}$ that depends (probably quadratically) on the dimension $d$.

\section{References}

1. M. Aigner and G. Ziegler, Proofs from The Book, 2nd edn., Springer-Verlag, Berlin, 2001.

2. A. Blokhuis and Á. Seress, The number of directions determined by points in the three-dimensional Euclidean space, Discrete Comput. Geom. 28 (2002), 491-494.

3. G. R. Burton and G. B. Purdy, The directions determined by $n$ points in the plane, J. London Math. Soc. (2) 20 (1979), 109-114.

4. R. Cordovil, The directions determined by $n$ points in the plane, a matroidal generalization, Discrete Math. 43 (1983), 131-137.

5. H. Edelsbrunner, Algorithms in Combinatorial Geometry, Springer-Verlag, Heidelberg, 1987.

6. P. Erdős, Solution to Problem Nr. 4065, Amer. Math. Monthly 51 (1944), 169.

7. J. C. Fisher and R. E. Jamison, Properties of affinely regular polygons, Geom. Dedicata 69 (1998), 241259.

8. J. E. Goodman and R. Pollack, A combinatorial perspective on some problems in geometry, Congr. Numer. 32 (1981), 383-394.

9. J. E. Goodman and R. Pollack, Allowable sequences and order types in discrete and computational geometry, in: New Trends in Discrete and Computational Geometry (J. Pach, ed.), Algorithms Combin. 10, Springer, Berlin, 1993, pp. 103-134.

10. R. E. Jamison, Survey of the slope problem, in: Discrete Geometry and Convexity, Ann. New York Acad. Sci. 440, New York Academy of Science, New York, 1985, pp. 34-51.

11. R. E. Jamison and D. Hill, A catalogue of sporadic slope-critical configurations. in: Proceedings of the Fourteenth Southeastern Conference on Combinatorics, Graph Theory and Computing (Boca Raton, Fla., 1983), Congr. Numer. 40, Utilitas Math., Winnipeg, 1983, pp. 101-125.

12. G. Korchmaros, Poligoni affin-regolari dei piani di Galois d'ordine dispari (English), Atti Accad. Naz. Lincei Rend. Cl. Sci. Fis. Mat. Natur. (8) 56 (1974), 690-697.

13. L. Lovász and A. Schrijver, Remarks on a theorem of Rédei, Studia Sci. Math. Hungar. 16 (1981), 449-454.

14. J. Pach, R. Pinchasi, and M. Sharir, On the number of directions determined by a three-dimensional point set, J. Combin. Theory Ser. A 108 (2004), 1-16.

15. L. Rédei, Lacunary Polynomials Over Finite Fields, North-Holland, Amsterdam, 1973.

16. P. R. Scott, On the sets of directions determined by $n$ points, Amer. Math. Monthly 77 (1970), 502-505.

17. T. Szőnyi, Around Rédei's theorem, Discrete Math. 208/209 (1999), 557-575.

18. P. Ungar, $2 N$ noncollinear points determine at least $2 N$ directions, J. Combin. Theory Ser. A 33 (1982), 343-347.

19. F. Wettl, On the nuclei of a pointset of a finite projective plane, J. Geom. 30 (1987), 157-163.

Received February 28, 2006, and in revised form October 2, 2006. Online publication July 13, 2007. 